RECEIVED

DOE/NV/10845-50

JAN 181995

OSTI

\title{
POTENTIAL HYDROLOGIC CHARACTERIZATION WELLS \\ IN AMARGOSA VALLEY
}

by

Brad Lyles and Todd Mihevc

SEPTEMBER 1994

Publication \#45129 
This report was prepared as an account of work sponsored by the United States Government. Neither the United States nor the United States Department of Energy, nor any of their employees, makes any warranty, express or implied, or assumes any legal liability or responsibility for the accuracy, completeness or usefulness of any information, apparatus, product or process disclosed, or represents that its use would not infringe privately owned rights. Reference herein to any specific commercial product, process, or service by trade name, mark, manufacturer, or otherwise, does not necessarily constitute or imply its endorsement, recommendation, or favoring by the United States Government or any agency thereof. The views and opinions of authors expressed herein do not necessarily state or reflect those of the United States Government or any agency thereof.

This report has been reproduced directly from the best available copy.

Available to DOE and DOE contractors from the Office of Scientific and Technical Information, P.O. Box 62, Oak Ridge, TN 37831; prices available from (615) 576-8401.

Available to the public from the National Technical Information Service, U.S. Department of Commerce, 5285 Port Royal Rd., Springfield, VA 22161. 


\section{DISCLAIMER}

Portions of this document may be illegible in electronic image products. Images are produced from the best available original document. 


\title{
POTENTIAL HYDROLOGIC CHARACTERIZATION WELLS IN AMARGOSA VALLEY
}

\author{
by \\ Brad Lyles and Todd Mihevc \\ Water Resources Center \\ Desert Research Institute \\ University and Community College System of Nevada
}

Publication No. 45129

prepared for

Nevada Operations Office

U.S. Department of Energy

Las Vegas, Nevada

September 1994

The work upon which this report is based was supported by the U.S. Department of Energy under Contract \#DE-AC08-90NV10845.

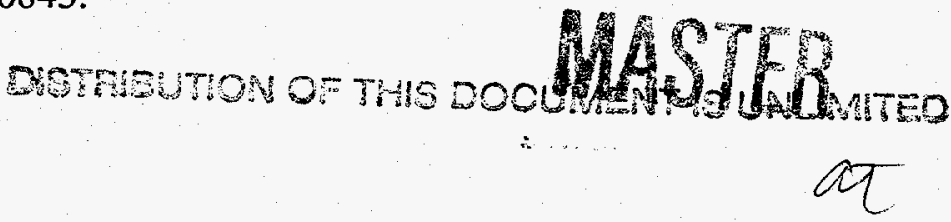




\begin{abstract}
More than 500 domestic, agricultural, and monitoring wells were identified in the Amargosa Valley. From this list, 80 wells were identified as potential hydrologic characterization wells, in support of the U.S. Department of Energy (DOE) Underground Test Area/Remedial Investigation and Feasibility Study (UGTA/RIFS). Previous hydrogeologic studies have shown that groundwater flow in the basin is complex and that aquifers may have little lateral continuity. Wells located more than $10 \mathrm{~km}$ or so from the Nevada Test Site (NTS) boundary may yield data that are difficult to correlate to sources from the NTS. Also, monitoring well locations should be chosen within the guidelines of a hydrologic conceptual model and monitoring plan. Since these do not exist at this time, recompletion recommendations will be restricted to wells relatively close (approximately $20 \mathrm{~km}$ ) to the NTS boundary.

Recompletion recommendations were made for two abandoned agricultural irrigation wells near the town of Amargosa Valley (previously Lathrop Wells), for two abandoned wildcat oil wells about $10 \mathrm{~km}$ southwest of Amargosa Valley, and for Test Well 5 (TW-5), about $10 \mathrm{~km}$ east of Amargosa Valley.
\end{abstract}




\section{CONTENTS}

ABSTRACT $\ldots \ldots \ldots \ldots \ldots \ldots \ldots \ldots \ldots \ldots \ldots \ldots \ldots \ldots \ldots \ldots \ldots \ldots \ldots$

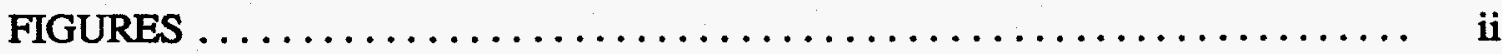

OBJECTIVE $\ldots \ldots \ldots \ldots \ldots \ldots \ldots \ldots \ldots \ldots \ldots \ldots \ldots \ldots \ldots \ldots \ldots \ldots \ldots \ldots \ldots \ldots \ldots$

INTRODUCTION $\ldots \ldots \ldots \ldots \ldots \ldots \ldots \ldots \ldots \ldots \ldots \ldots \ldots \ldots$

AVAILABLE WELLS IN AMARGOSA VALLEY $\ldots \ldots \ldots \ldots \ldots \ldots \ldots \ldots$

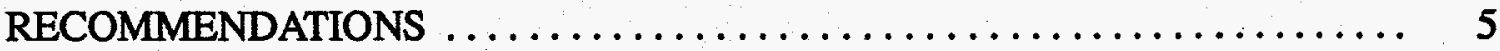

Abandoned Irrigation Wells $\ldots \ldots \ldots \ldots \ldots \ldots \ldots \ldots \ldots \ldots \ldots \ldots \ldots \ldots$

Abandoned Oil Wells $\ldots \ldots \ldots \ldots \ldots \ldots \ldots \ldots \ldots \ldots \ldots \ldots \ldots \ldots \ldots$

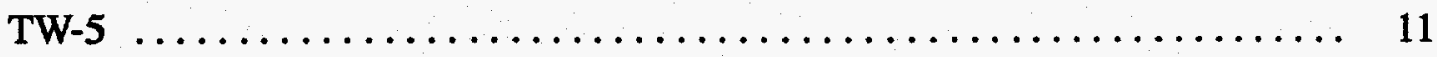

CONCLUSIONS $\ldots \ldots \ldots \ldots \ldots \ldots \ldots \ldots \ldots \ldots \ldots \ldots \ldots \ldots \ldots \ldots \ldots \ldots \ldots \ldots$

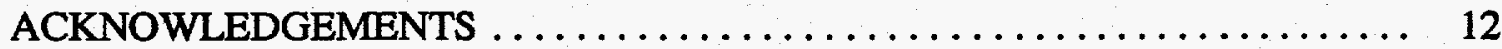

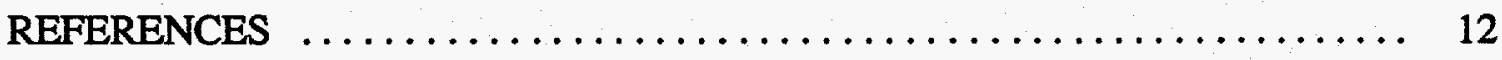

APPENDIX A: 613 Wells Located in Amargosa Valley .............. A-1

APPENDIX B: 80 Potential Hydrologic Characterization Recompletion Wells ..... B-1

\section{FIGURES}

1. Location map for the Nevada Test Site $\ldots \ldots \ldots \ldots \ldots \ldots \ldots \ldots \ldots$

1. General location map of Amargosa Valley $\ldots \ldots \ldots \ldots \ldots \ldots \ldots \ldots \ldots$

2. Location map of potential groundwater characterization wells in Amargosa Valley

(1)

3. Generalized well completion schematic of abandoned agricultural well site 83.

4. Generalized well completion schematic of abandoned agricultural well site 90.

5. Generalized well completion schematic for Test Well-5 (TW-5). . . . . . . 8

6. Generalized well completion schematic of Federhoff-Federal $5-1 \ldots \ldots \ldots \ldots .9$

7. Generalized well completion schematic of Federhoff-Federal 25-1........ 10 


\section{OBJECTIVE}

Existing wells are identified that may be used to characterize the hydrogeologic conditions of Amargosa Valley. These wells include active and abandoned agricultural, domestic, and monitoring wells. Hydrologic data from these wells will be used to develop a conceptual model of the hydrogeology downgradient of the Nevada Test Site (NTS), in support of the Underground Test Area Remedial Investigation and Feasibility Study (UGTA/RIFS) program. This report includes a brief review of the hydrologic literature related to the Amargosa Valley and vicinity, a list of wells that could be evaluated in the future, and a list of recommendations for the recompletion of wells near the NTS boundary.

\section{INTRODUCTION}

Past hydrogeologic studies were conducted to evaluate the water-resources potential of the area (Malmberg and Eakin, 1962; Walker and Eakin, 1963), evaluate the impacts of groundwater pumping (Dudley and Larson, 1976), estimate groundwater recharge from wash infiltration (Osterkamp et al., 1994), and evaluate regional groundwater flow (Rush, 1971; Blankennagal and Weir, 1973; Winograd and Thordarson, 1975; Claassen, 1975; Czarnecki and Waddell, 1994; Waddell et al., 1984; Bedinger et al., 1989; Harrill et al., 1988; Dettinger, 1989; Burbey and Prudic, 1991). Specific hydrologic studies were conducted by Akers (1974) to evaluate the hydrologic relation of groundwater in the Amargosa Desert to spring discharge in Death Valley. Groundwater levels in the Amargosa Desert were most recently evaluated by Kilroy (1991) and a report is currently being drafted on the hydrogeology and geochemistry of the area (Czarnecki, 1994, personal communication).

The evapotranspiration rate in the area is high, particularly in the summer, because of the low humidity and the high temperatures. Studies conducted in the area by Nichols (1987) suggest that nearly $1.9 \mathrm{~m}$ of water are lost to evapotranspiration annually.

The geology of the area is complex, both lithologically and structurally. The oldest geologic units in the area are paleozoic rocks of various lithologies: carbonate (limestone and dolomite) and non-carbonate (shale, quartzite, siltstone, etc.). These units are depositionally overlain by Tertiary volcanic ash-fall tuff, sedimentary valley-fill, playa, and lakebed deposits, and Quaternary alluvium. Several major faults have been mapped in the area which in some cases may disrupt lateral continuity of aquifers or in other areas may form conduits for groundwater flow.

Springs and seeps in the area issue primarily from carbonate-rock aquifers and to a lesser extent from non-carbonate aquifers. Water from the Paleozoic aquifers are generally from regional groundwater flow systems (Mifflin, 1968; Dettinger, 1989). Previous studies suggest that regional groundwater flow systems may be interconnected from Death Valley to the White River flow system, some $483 \mathrm{~km}$ northeast of Death Valley.

Most groundwater is pumped from valley-fill and alluvial aquifers overlying the bedrock aquifers. The impact of pumping in the sedimentary aquifers on the underlying carbonate aquifers 
is poorly understood. At Ash Meadows, direct connections between pumping from the alluvial aquifers and water level declines in the carbonate rocks were demonstrated. Groundwater development near Devil's Hole lowered water levels by more than $30 \mathrm{~cm}$ in the carbonate aquifers between 1969-72 (Bateman et al., 1974; Dudley and Larson, 1976). The water levels recovered slowly over a period of about 15 years after pumping ceased. A study conducted by Akers (1974) showed that heavy pumping in the Amargosa hydrologic basin may produce decreased spring flow in Death Valley in 10 to 40 years.

Only small amounts of surface water are found within the area. Several small springs exist near Beatty, in the Amargosa Desert and in Death Valley. Discharge from the springs varies from a seep to over 378 liters per minute, all of which either is collected for consumptive use or flows a short distance before percolating into sedimentary units and/or being evapotranspired. Additionally, several small ponds exist in Amargosa Valley at the locations of borrow pits, where the water table has been intersected. Runoff from intensive precipitation may occur for short periods of time in washes or streambeds in the area.

\section{AVAILABLE WELLS IN AMARGOSA VALLEY}

Upon review of the literature, the most extensive inventories of wells in the area were contained in two U.S. Geological Survey (USGS) reports (Thordarson et al., 1967; Thordarson and Robinson, 1971; Kilroy, 1991). For this study, a database of approximately 500 wells was constructed from a USGS database. Wells north of $36^{\circ} 40^{\prime} 23^{\prime \prime}$ and east of $116^{\circ} 29^{\prime} 46^{\prime \prime}$ (within the NTS) were removed from the database. The remaining 611 records are listed in Appendix A. Two "wild cat" oil wells in Amargosa Valley were also added to Appendix A. Of the 613 records, there were 526 wells (i.e., 87 wells have duplicate entries with the same location). All 526 wells are shown on the location map (Figure 1).

Eight wells (Army Well 1, Army 6, Army 6A, Camp Desert Rock, and four wells east of Yucca Mountain), were excluded from the list, as these are considered part of the "on-site" DOE programs. An additional 34 shallow monitoring wells are associated with the U.S. Ecology disposal facility and will not be further evaluated. The remaining wells are sparsely distributed between Beatty and Pahrump, with clusters of wells near Pahrump and the area southwest of the town of Amargosa Valley.

Most of the wells in Amargosa Valley are shallow (15 to $30 \mathrm{~m}$ deeper than the water table). The scope of this study was to identify wells that may used to help develop a conceptual model of the regional hdyrogeology. Therefore, wells near the NTS, where sparse data exist, and wells that penetrate more than $50 \mathrm{~m}$ of saturated interval, were deemed potential hydrologic characterization wells. Based on these criteria, 80 wells were identified as potential hydrologic characterization wells (Figure 2, Appendix B).

Many of these wells are U.S. Park Service monitoring wells and are shown as being owned by the "USGS" or "US BORAX" in Appendix B. These wells primarily parallel the California/Nevada 


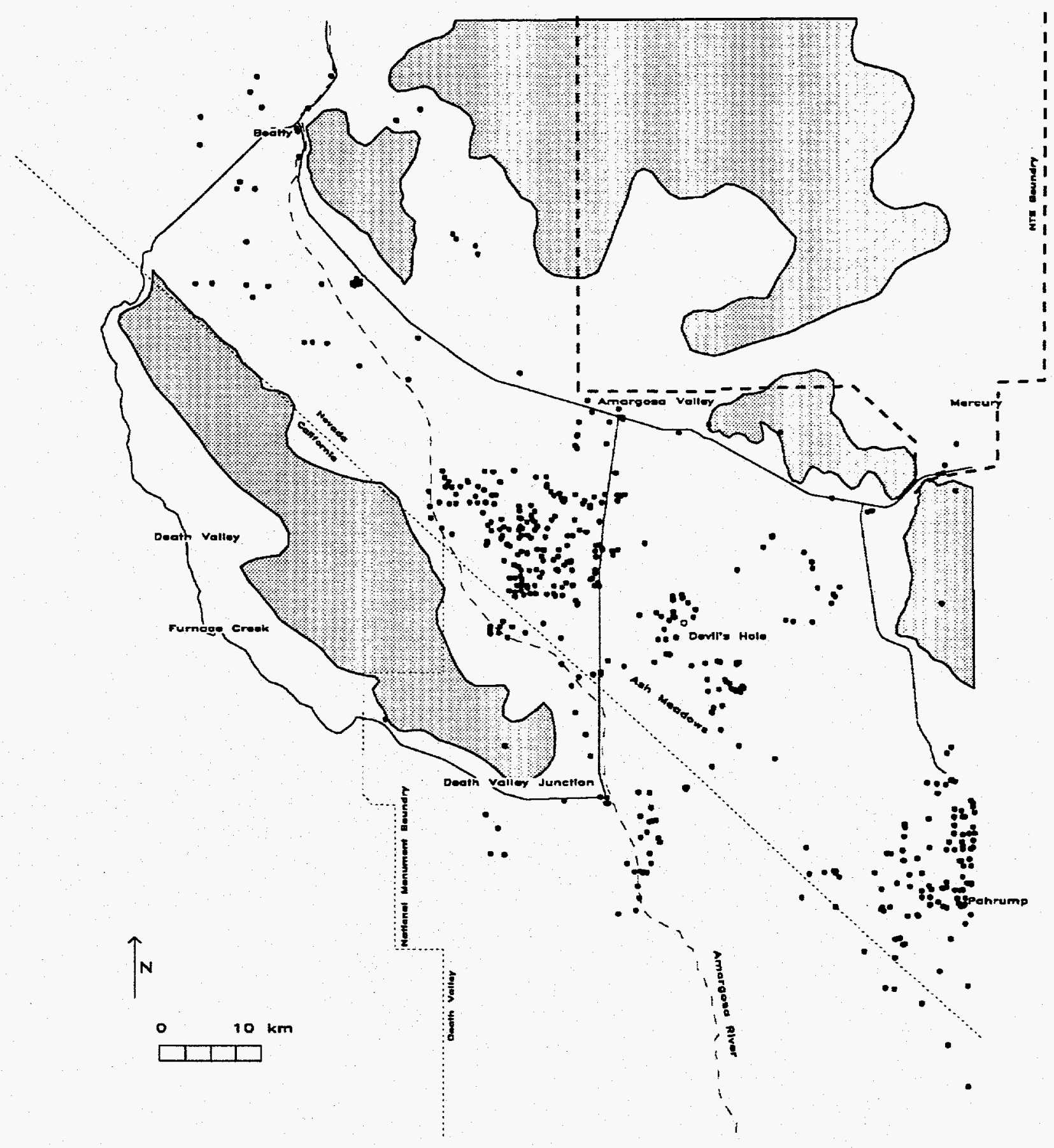

Figure 1. General location map of Amargosa Valley (526 wells are represented with dots, bed-rock areas are shaded). 


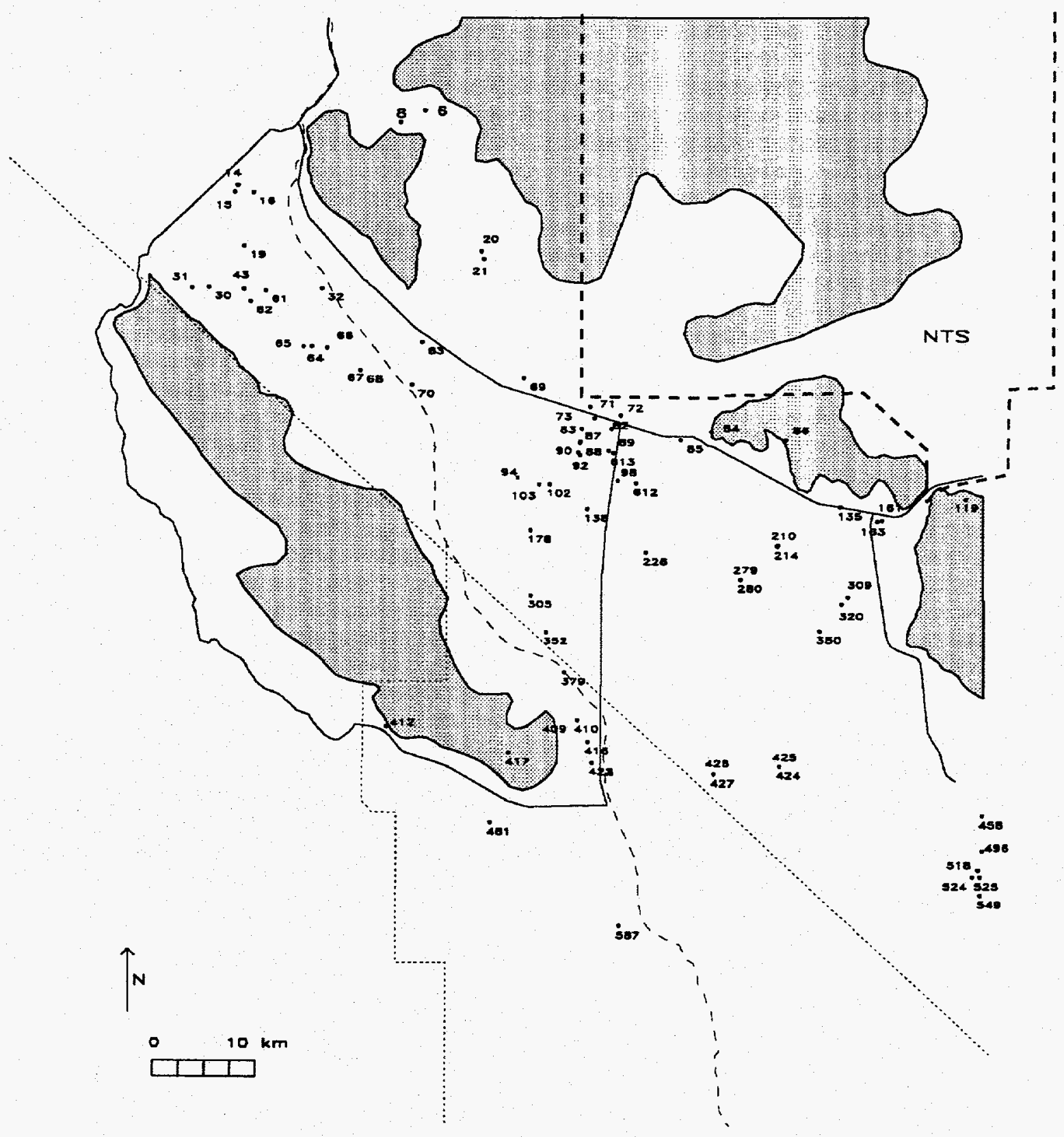

Figure 2. Location map of potential groundwater characterization wells in Amargosa Valley $(80$ wells are represented with dots, numbers correspond to site numbers in the appendices, and bed-rock areas are shaded). 
border from Beatty to Site 68 (Figure 2), and are completed at various depths, ranging from 98 to $762 \mathrm{~m}$. Most of these wells were drilled specifically for the purpose of monitoring and therefore do not require recompletion.

Sites 6 and 8 are located between the NTS and Beatty and are in the approximate location of a gold mining operation. These wells are believed to be currently used for construction water; however, this area has not yet been field checked. Information on the lithology is not available for these wells.

Sites 71, 83, 87 and 90 are abandoned agricultural irrigation wells near the town of Amargosa Valley. These sites were visited in 1993 and their locations were confirmed with a global positioning system unit. Site 71 had an original total depth (TD) of $295 \mathrm{~m}$ and now has bottom-hole depth of $55 \mathrm{~m}$. Site 83 currently has a shaft-driven pump installed with the motor removed. This well was drilled to a TD of $174 \mathrm{~m}$ and in 1987 had a depth to water of $90.1 \mathrm{~m}$ (Figure 3). The current depth to water is unknown. Site 87 originally had a TD of $152 \mathrm{~m}$ and in $1962 \mathrm{had}$ a depth to water of 77.8 $\mathrm{m}$. This well is currently dry. Site 90 (site 92 is thought to be the same well) was drilled to a TD of $135 \mathrm{~m}$ and in 1987 had a depth to water of $71.7 \mathrm{~m}$ (Figure 4). This well is currently open and has a depth to water of $72.1 \mathrm{~m}$. These irrigation wells are all constructed with $30+\mathrm{cm}$-diameter casing, which may be ample size for most recompletion projects.

Site 84 (TW-5, hydrologic test well 5) was drilled by the Atomic Energy Commission in 1962 to TD of $279 \mathrm{~m}$ (West and Garber, 1962). The well was completed with 33.8-cm-diameter conductor (cemented from land surface to $51 \mathrm{~m}$ deep) and $17.8-\mathrm{cm}$-diameter casing from 0 to $244 \mathrm{~m}$ deep (the bottom $19 \mathrm{~m}$ were machine-cut vertical-slot casing) (Figure 5). The bottom $230 \mathrm{~m}$ were completed in Paleozoic carbonate rocks of the Carrara Formation, which were overlain by Tertiary alluvium. The most recent water level measurement was made in 1962 and was $205.4 \mathrm{~m}$ below land surface. The road to the site has been eroded and is currently impassible.

Sites $86,135,161$ and 163 could not be found during field investigations and are believed to have been destroyed.

Two abandoned exploration oil wells are located approximately $6 \mathrm{~km}$ southwest of Amargosa Valley town: Federhoff-Federal 5-1 and Federhoff-Federal 25-1 (hereon referred to as 5-1 and 25-1, respectively). These wells were drilled in September-November 1991. Well 5-1 was drilled to a total depth of $447 \mathrm{~m}$ and was abandoned by setting three cement bridge plugs with heavy drilling mud between the plugs (Figure 6). The upper $259 \mathrm{~m}$ of the hole penetrated Neogene(?) deposits followed by carbonate rocks (Harris et al., 1992). Well $25-1$ was drilled to a total depth of $1530 \mathrm{~m}$ and was also abandoned by setting three cement bridge plugs with heavy mud between the plugs (Figure 7). The upper $671 \mathrm{~m}$ of the hole penetrated Neogene(?) deposits followed by $305 \mathrm{~m}$ of Paleozoic carbonate rocks of the Nopah Formation; the remainder of the hole is Goodwin Limestone (Harris et al., 1992). These wells were abandoned in such a way that they could be recompleted as monitoring wells. The Yucca Mountain Project originally intended to complete these as monitoring wells, but has lost interest in them in the last two years (Czarnecki, 1994, personal communication). Therefore, the wells are available for recompletion as UGTA/RIFS wells. 


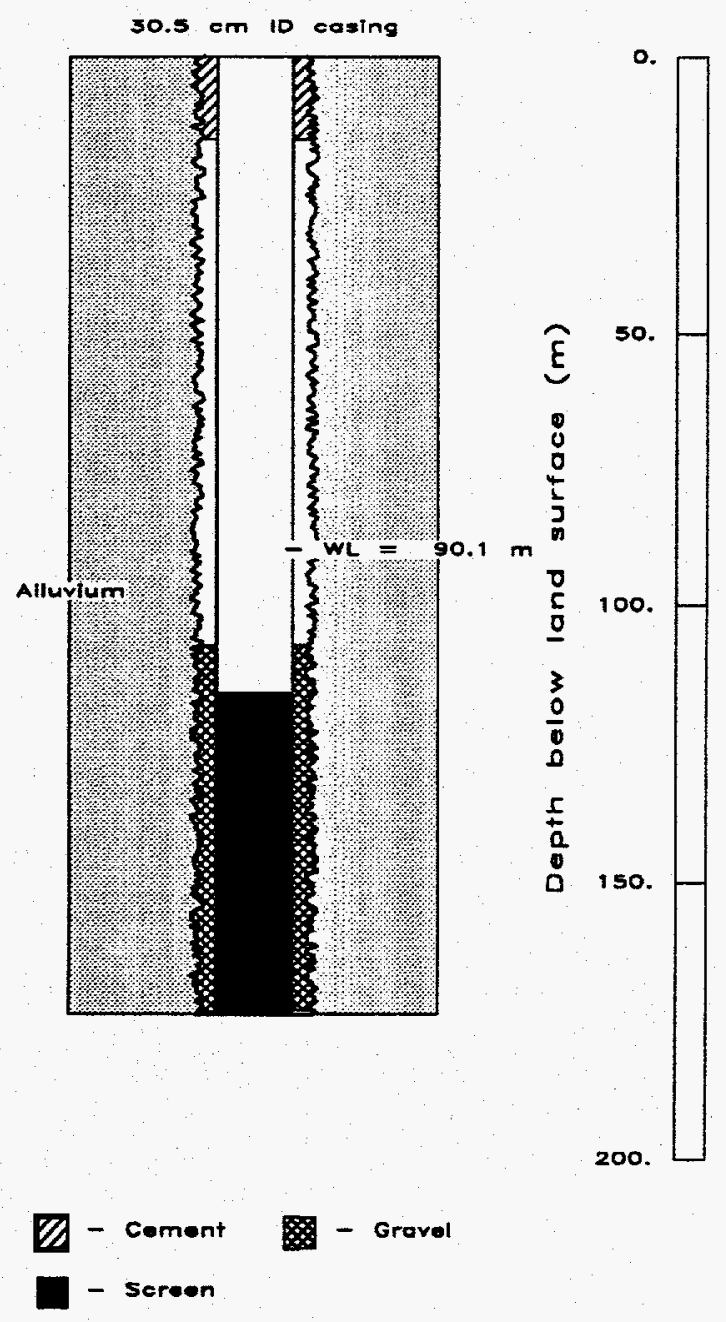

Figure 3. Generalized well completion schematic of abandoned agricultural well site 83. 


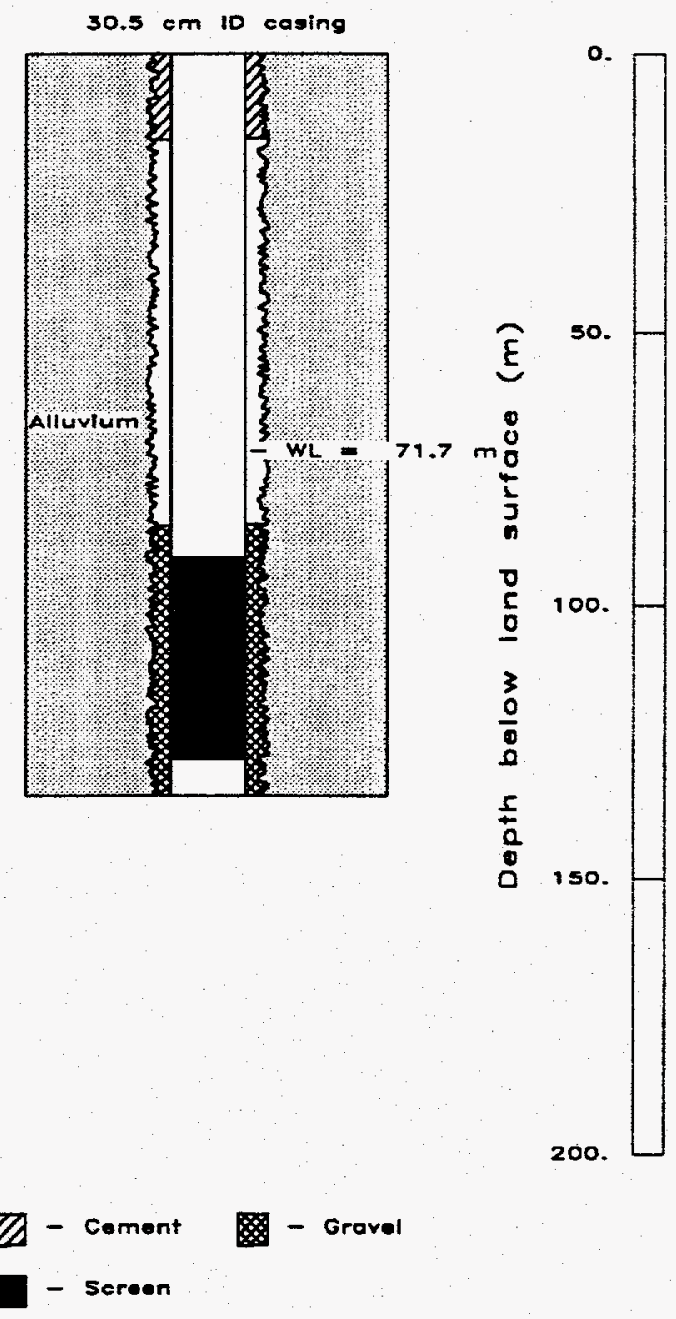

Figure 4. Generalized well completion schematic of abandoned agricultural well site 90 . 


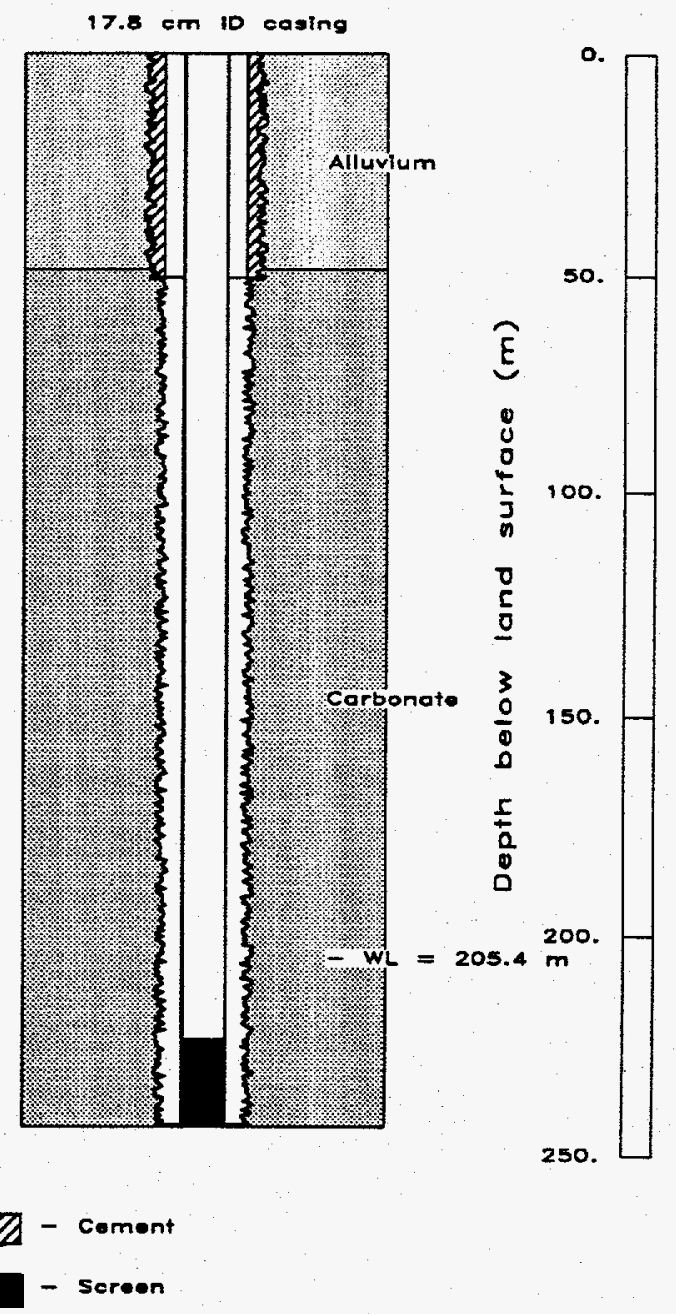

Figure 5. Generalized well completion schematic for Test Well-5 (TW-5). 


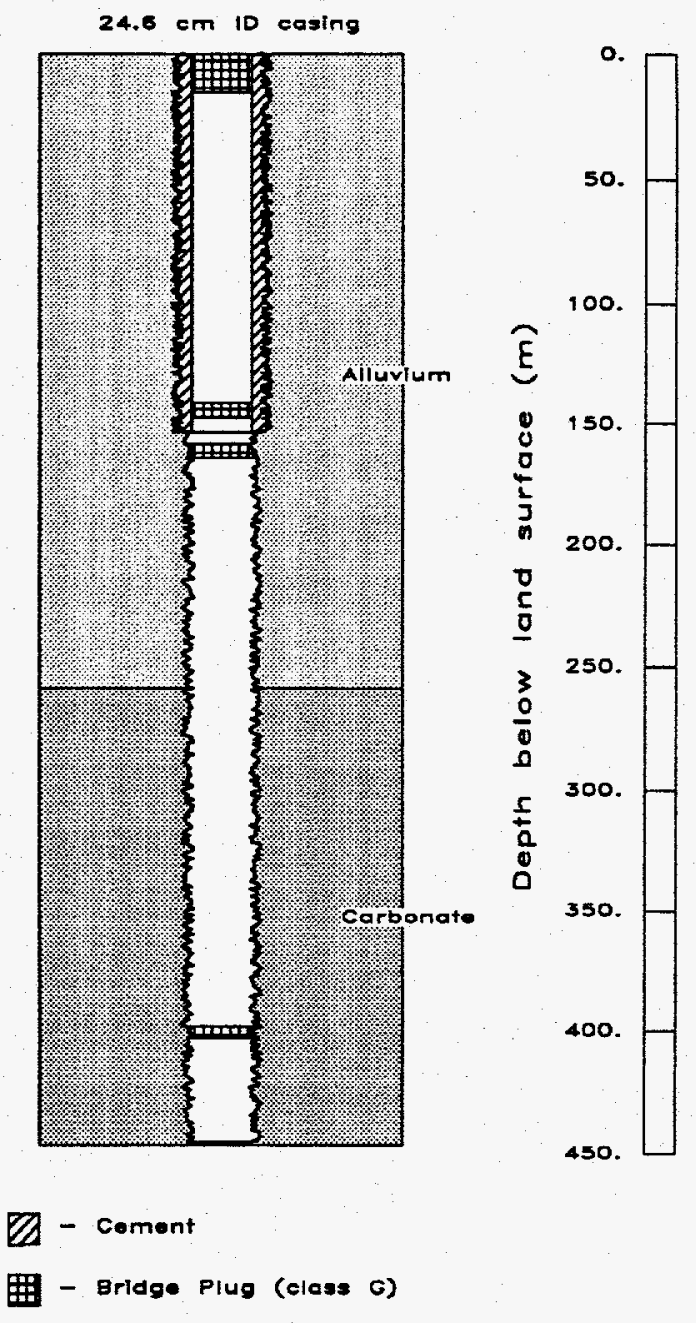

Figure 6. Generalized well completion schematic of Federhoff-Federal 5-1. 


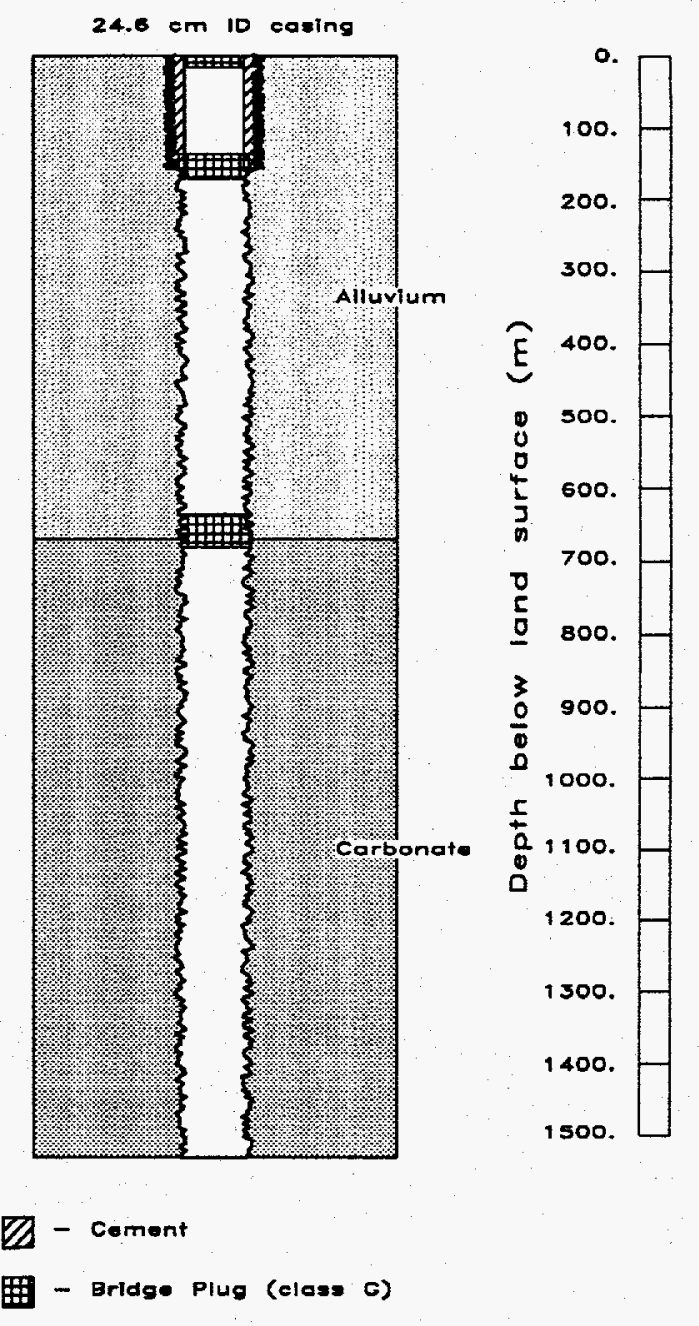

Figure 7. Generalized well completion schematic of Federhoff-Federal 25-1. 


\section{RECOMMENDATIONS}

The abandoned agricultural wells near Amargosa Valley, the two abandoned oil wells, and well TW-5 appear to be the most suitable wells for recompletion as monitoring wells, based on their proximity to the NTS boundary, their downgradient hydrologic location to the NTS, and their current ownership/use. If the agricultural wells are truly abandoned, it may be possible for the Nevada State Engineer to condemn the wells and donate them to the DOE for recompletion. The oil wells and well TW-5 are currently owned by the U.S. government (Bureau of Land Management and DOE, respectively); therefore, gaining access to these wells should not be a problem.

These steps are required for the sites to be included in the UGTA/RIFS program:

\section{Abandoned Irrigation Wells}

- Establish ownership of the wells

- Remove the pump from site 83 well

- Secure well heads with locking steel plates

- Perform basic casing integrity logs and video logs

- Install pumps in sites 83 and 90 and perform single-well aquifer tests, compute hydraulic parameters

- Establish a monitoring program based on program needs

\section{Abandoned Oil Wells}

- Change ownership of the wells from BLM to DOE

- Review hole history

- Develop well completion schedule, drilling plan, etc.

- Build drill pad, locate well casings

- Drill out cement bridge-plugs

- Install single completion monitoring well string or sliding-sleeve casing

- Develop well and perform aquifer test if possible

- Establish a monitoring program based on program needs

TW-5

- Establish access permission from Nevada Department of Transportation

- Evaluate road condition, repair as needed

- Secure well head with locking steel plate

- Perform basic casing integrity log and video log

- Install a pump and perform single-well aquifer tests, compute hydraulic parameters

- Establish a monitoring program based on program needs 


\section{CONCLUSIONS}

Of the 524 wells in Amargosa Valley, 80 were identified as potential hydrologic characterization wells. Five wells within a $10-\mathrm{km}$ radius of the town of Amargosa Valley were discussed and recommendations were made related to recompletion strategies. Based on previous experience drilling monitoring wells on the NTS, recompleting existing wells may save as much as 40 percent when compared to spudding new holes. Of the five wells described in the recommendation section, the work at TW-5 would require the least work and therefore may be the least expensive, followed by the abandoned agricultural wells, and finally the abandoned wildcat wells. Although the wildcat wells may be the most expensive to recomplete, they intersect more saturated interval and would yield valuable information about the regional carbonate aquifer.

It is recommended that a recompletion schedule be prioritized as follows:

1. TW-5

2. wildcat wells

3. agricultural wells

\section{ACKNOWLEDGEMENTS}

The authors would like to thank Randy Laczniak and Dave Wood of the U.S. Geological Survey for their help in developing the database for this report.

\section{REFERENCES}

Akers, J.P., 1974, "The relation of groundwater flow in Amargosa Desert, California-Nevada, to spring discharge in Death Valley California." U.S. Geological Survey Administrative Report, Menlo Park, CA, pp 9.

Bateman, M.S., Mindling, A.L. and Naff, R.L., 1974, "Development and management of groundwater in relation to preservation of desert pupfish in Ash Meadows, southern Nevada." University of Nevada, Desert Research Institute, Pub. No. 17, pp 39.

Bedinger, M.S., J.R. Harrill, W.H. Langer, J.M. Thomas and D.A. Mulvihill, 1984, "Maps showing ground water levels, springs, and depth to groundwater, basin and range province, Nevada."U.S. Geological Survey Water-Resources Investigation 83-4119-B.

Bedinger, M.S., K.A. Sargent and W.H. Langer, 1989, "Studies of the geology and hydrology in the basin and range province, southwestern United States, for isolation of high-level radioactive waste - characterization of the Death Valley region, Nevada and California." U.S. Geological Survey Professional Paper 1370-F, pp 35.

Blankennagel, R.K. and J.E. Weir, 1973, "Geohydrology of the eastern part of Pahute Mesa, Nevada Test Site, Nye County, Nevada.” U.S. Geological Survey Professional Paper 712-B, pp 35. 
Burbey, T.J. and Prudic, D.E., 1991, "Conceptual evaluation of regional groundwater flow in the carbonate-rock province of the Great Basin, Nevada, Utah, and adjacent states." U.S. Geological Survey Professional Paper 1409-D, pp 84.

Claassen, H.C., 1975, "Sources and mechanisms of recharge for groundwater in west-central Amargosa Desert, Nevada -- a geochemical interpretation." U.S. Geological Survey Professional Paper 712-F, pp 30.

Czarnecki, J.B. and R.K., Waddell, 1984, "Finite-element simulation of ground water flow in the vicinity of Yucca Mountain, Nevada-California." U.S. Geological Survey Water-Resources Investigations Report 84-4349, pp38.

Dettinger, M.D., 1989, "Distribution of carbonate rock aquifers in southern Nevada and the potential for their development, summary of findings, 1985-88." Summary Report 1, U.S. Geological Survey and Desert Research Institute, pp 37.

Dudley, W.W., Jr. and J.D. Larson, 1976, "Effect of irrigation pumping on desert pupfish habitats in Ash Meadows, Nye County, Nevada." U.S. Geological Survey Professional Paper 927, pp 52.

Grove, D.B., 1971, "U.S. Geological Survey tracer study, Amargosa Desert, Nye County, Nevada; Part II: an analysis of the flow field of a discharging-recharging pair of wells." U.S. Geological Survey, USGS-474-99, pp 56.

Harrill, J.R., J.S. Gates and J.M. Thomas, 1988, "Major ground water flow systems in the Great Basin region of Nevada, Utah, and adjacent states." U.S. Geological Survey, Hydrologic Atlas, 694-C.

Harris, A.G., J.E. Repetski, J. Clayton, J.A. Grow, M.D. Carr and T.A. Daws, 1992, "Results from 1991 wildcat wells near Yucca Mountain, Nevada." Geological Society of America, Abstracts with Programs, Vol. 24, No. 6.

Kilroy, K.C., 1991, "Groundwater conditions in Amargosa Desert, Nevada-California, 1952-87." U.S. Geological Survey Water-Resources Investigations Report 89-4101, pp 93.

Malmberg, G.T. and T.E. Eakin, 1962, "Groundwater appraisal of Sarcobatus Flat and Oasis Valley, Nye and Esmeralda Counties, Nevada." State of Nevada, Department of Conservation and Natural Resources, Groundwater Reconnaissance Series, report 10, pp 39.

Mifflin, M.D., 1968, "Delineation of groundwater flow systems in Nevada." Desert Research Institute, Water Resources Center, Technical Report Series H-W, No. 4, pp 111.

Nichols, W.D., 1987, "Geohydrology of the unsaturated zone at the burial site for low-level radioactive waste near Beatty, Nevada, Nye County, Nevada." U.S. Geological Survey Water-Supply Paper 2312, pp 52.

Osterkamp, W.R., L.J. Lane and C.S. Savard, 1994, "Recharge estimates using a geomorphic/ distributed-parameter simulation approach, Amargosa River Basin." Water Resources Bulletin, Vol 30, No. 3, pg 493-507. 
Rush, F.E., 1971, "Regional groundwater systems in the Nevada Test Site area, Nye, Lncoln, and Clark Counties, Nevada." State of Nevada, Department of Conservation and Natural Resources, Water Resources Reconnaissance Series, Rpt. No. 54, pp 25.

Thordarson, W., R.A. Young and I.J. Winograd, 1967, "Records of wells and test holes in the Nevada Test Site and vicinity (through December 1966); U.S. Geological Survey, TEI-872, pp 26.

Thordarson, W. and B.P. Robinson, 1971, "Wells and springs in California and Nevada within 100 miles of the point $37^{\circ} 15^{\prime}$ N., $116^{\circ} 25^{\prime}$ W., on the Nevada Test Site." U.S. Geological Survey, USGS-474-85, pp 178.

Walker, G.E. and T.E. Eakin, 1963, "Geology and groundwater of Amargosa Desert, Nevada-California." State of Nevada, Department of Conservationandf Natural Resources, Groundwater Reconnaissance Series, report 14, pp 45.

Waddell, R.K., J.H. Robinson and R.K. Blankennagel, 1984, "Hydrology of Yucca Mountain and vicinity, Nevada-California--investigation results through mid-1983." U.S. Geological Survey, Water-Resources Investigations Report 84-4267, pp 72.

West, L.R. and M.S. Garber, 1962, "Summary of hydraulic tests and abridged lithologic log for groundwater test well 5, Nye County, Nevada." U.S. Geological Survey Technical Letter: NTS-34, pp 7.

Winograd, I.J. and W. Thordarson, 1975, "Hydrogeologic and geochemical framwork, south-central Great Basin with special reference to the Nevada Test Site." U.S. Geological Survey Professional Paper 712-C, pp 126. 


\section{APPENDIX A}

\section{WELLS LOCATED IN AMARGOSA VALLEY}

Appendix water level codes under the heading descriptor:

$$
\begin{aligned}
& O \text { - original } \\
& C \text { - current }
\end{aligned}
$$

Codes:

$$
\begin{aligned}
& \text { D - dry } \\
& \text { E - recently flowing } \\
& F \text { - flowing } \\
& \text { G - nearby site flowing } \\
& H \text { - nearby site recently flowing } \\
& I \text { - injection of water } \\
& \text { J - nearby site injected with water } \\
& \text { N - measurement discontinued } \\
& O \text { - obstruction above water surface } \\
& P \text { - pumping } \\
& R \text { - recently pumped } \\
& S \text { - nearby site pumping } \\
& T \text { - nearby site recently pumping } \\
& V \text { - foreign substance on water surface } \\
& W \text { - well destroyed } \\
& X \text { - surface-water affects } \\
& Z \text { - other (explain in remarks) }
\end{aligned}
$$

Note: Latitude and Longitude (deg, $\mathrm{min}, \mathrm{sec}$ )

Elevation ( $m$ above mean sea level)

Total Depth, Perforation Interval and Water Level ( $\mathrm{m}$ below land surface)

Date (month/day/year) 


\begin{tabular}{|c|c|c|c|c|c|c|c|c|c|c|}
\hline $\begin{array}{l}\text { Site } \\
\text { No }\end{array}$ & Lat & Long & $\begin{array}{l}\text { Land } \\
\text { Elev }\end{array}$ & $\begin{array}{l}\text { Total } \\
\text { Depth }\end{array}$ & $\begin{array}{l}\text { Perfo } \\
\text { top }\end{array}$ & $\begin{array}{l}\text { ration } \\
\text { bottom }\end{array}$ & $\begin{array}{l}\text { Water } \\
\text { Level }\end{array}$ & $\begin{array}{l}\text { Measure } \\
\text { Date }\end{array}$ & $\begin{array}{l}\text { Note } \\
\text { O C }\end{array}$ & Site Name \\
\hline 1 & 365711 & 1643051 & 097.2 & & & & & $00-00-00$ & & \\
\hline 2 & 365709 & 1164811 & 1292.3 & 211.2 & 61.0 & 210.3 & 53.9 & $08-22-88$ & & BEATTY INDIAN SPR WELL \\
\hline 3 & 365619 & 1164839 & 1286.2 & & & & & $00-00-00$ & & BEATTY MIDDLE WELL \\
\hline 4 & 365527 & 1164753 & 1170.4 & 213.3 & 73.1 & 213.3 & 9.1 & $01-15-89$ & & BEATTY SUMMIT WELL \\
\hline 5 & 365524 & 1164440 & 1097.2 & 61.0 & & & 29.0 & $00-00-00$ & & \\
\hline 6 & 365520 & 1163703 & 1198.1 & 487.7 & 243.8 & 487.7 & 201.2 & 09-01-89 & & GEXA WELL 4 \\
\hline 7 & 365457 & 1165158 & 1341.1 & 53.3 & & & 24.4 & $00-00-00$ & & \\
\hline 8 & 365445 & 1163839 & 1243.8 & 213.3 & 63.4 & 213.3 & 41.1 & $09-18-88$ & & GEXA WELL 3 \\
\hline 9 & 365420 & 1164530 & & 91.4 & & & & $00-00-00$ & & BEATTY P.S. WELL \\
\hline 10 & 365409 & 1164523 & 1158.2 & 59.4 & & & 6.1 & $00-00-00$ & & \\
\hline 11 & 365326 & 1165202 & 1164.3 & 121.9 & & & 83.1 & $01-06-87$ & & \\
\hline 12 & 365247 & 1164518 & 969.2 & 37.8 & 9.1 & 36.9 & 5.4 & $01-15-87$ & & \\
\hline 13 & 365245 & 1164518 & & & & & & $00-00-00$ & & \\
\hline 14 & 365128 & 1164920 & & & & & & $00-00-00$ & & ETH I-4 \\
\hline 15 & 365106 & 1164932 & 364.2 & & & & 219.1 & $08-10-88$ & & II-1 \\
\hline 16 & 365105 & 1164819 & & & & & & $00-00-00$ & & II-2 \\
\hline 17 & 364837 & 1163452 & 978.4 & & & & 141.3 & $03-10-85$ & & USW VH-2 \\
\hline 18 & 364821 & 1163437 & 974.4 & 1219.1 & 219.4 & 1219.1 & 164.0 & $04-27-83$ & & USW VH-2 \\
\hline 19 & 364814 & 1164854 & 957.0 & 457.2 & 152.4 & 457.2 & 165.9 & $01-11-87$ & & \\
\hline 20 & 364758 & 1163317 & 976.8 & & & & 187.4 & $03-10-85$ & & USW VH-1 \\
\hline 21 & 364732 & 1163307 & 963.5 & 762.3 & 277.7 & 762.3 & 184.1 & $12-23-93$ & $\mathbf{z}$ & USW VH-1 \\
\hline 22 & 364615 & 1164124 & 848.5 & 93.6 & 85.9 & 92.0 & 87.3 & $05-15-89$ & & MW 313 \\
\hline 23 & 364615 & 1164124 & 848.4 & 145.4 & 140.5 & 143.9 & 92.7 & $10-10-90$ & & U.S. ECOLOGY - MW600 \\
\hline 24 & 364607 & 1164107 & 846.4 & 91.1 & 83.5 & 89.6 & 86.5 & $05-15-89$ & & MW 310 \\
\hline 25 & 364603 & 1164121 & 847.0 & 91.7 & 84.1 & 90.2 & 86.7 & $05-15-89$ & & MW 312 \\
\hline 26 & 364603 & 1164108 & 845.7 & 92.7 & 85.0 & 91.1 & 88.5 & $05-15-89$ & & MW 316 \\
\hline 27 & 364601 & 1164141 & 848.3 & 110.0 & 103.9 & 110.0 & 103.9 & $10-10-90$ & & U.S. ECOLOGY-W001 \\
\hline 28 & 364600 & 1164109 & 844.8 & 98.8 & 90.8 & 96.9 & 92.3 & $05-15-89$ & & MW 308 \\
\hline 29 & 364600 & 1164120 & 846.1 & 97.8 & 90.2 & 96.3 & 92.0 & $05-15-89$ & & MW 314 \\
\hline 30 & 364600 & 1165112 & 1011.9 & 127.4 & & & & $01-11-87$ & D & \\
\hline
\end{tabular}

Owner

BEATTY WATER AND SEWER
BEATTY WATER AND SEWER
BEATTY WATER AND SEWER

USNGS GEXA

GOLD GEXA

FARRAGUT

RANCHERS EQUIP \& SUPPLY COMPANY

USGS-WRD

U.S. GOVT

USGS

USGS-WRD

U.S. GOVT

US ECOLOGY

U.S. ECOLOGY

US ECOLOGY

US ECOLOGY

US ECOLOGY

U.S. BCOLOGY

US ECOLOGY

US ECOLOGY

US BORAX 


\begin{tabular}{|c|c|c|c|c|c|c|c|c|c|c|c|}
\hline $\begin{array}{l}\text { Site } \\
\text { No }\end{array}$ & Lat & Long & $\begin{array}{l}\text { Land } \\
\text { Elev }\end{array}$ & $\begin{array}{l}\text { Total } \\
\text { Depth }\end{array}$ & $\begin{array}{l}\text { Perfo } \\
\text { top }\end{array}$ & $\begin{array}{l}\text { ation } \\
\text { bottom }\end{array}$ & $\begin{array}{l}\text { Water } \\
\text { Level }\end{array}$ & $\begin{array}{l}\text { Measure } \\
\text { Date }\end{array}$ & $\begin{array}{l}\text { Note } \\
\text { O C }\end{array}$ & Site Name & Owner \\
\hline 31 & 364559 & 1165219 & 1146.0 & 144.8 & & & 144.6 & $10-30-86$ & & & US BORAX \\
\hline 32 & 364557 & 1164345 & 859.5 & 122.5 & 116.4 & 122.5 & 103.0 & $08-21-83$ & & & USGS \\
\hline 33 & 364557 & 1164114 & 844.4 & 99.1 & 91.4 & 97.5 & 92.5 & $05-15-89$ & & MW 311 & US ECOLOGY \\
\hline 34 & 364557 & 1164115 & 844.2 & 131.1 & 126.5 & 129.5 & 98.1 & $10-10-90$ & & U.S. ECOLOCY - MW604 & U.S. ECOLOGY \\
\hline 35 & 364557 & 1164109 & 844.6 & 99.4 & 91.7 & 97.8 & 93.1 & $05-15-89$ & & MW 309 & US ECOLOGY \\
\hline 36 & 364557 & 1164111 & 844.4 & 99.4 & 91.7 & 97.8 & 93.1 & $05-15-89$ & & MW 317 & US ECOLOGY \\
\hline 37 & 364557 & 1164118 & 844.7 & 99.4 & 91.7 & 97.8 & 92.8 & $05-15-89$ & & MW 315 & US ECOLOGY \\
\hline 38 & 364557 & 1164112 & 849.7 & 175.3 & & & 85.9 & $07-12-62$ & & & NUCLEAR ENGIN \\
\hline 39 & 364557 & 1164130 & 847.3 & 175.3 & 138.1 & 174.6 & 96.0 & $06-27-61$ & & & US ECOLOGY \\
\hline 40 & 364557 & 1164130 & 847.3 & 175.3 & 138.1 & 174.6 & & $01-07-87$ & $\mathbf{P}$ & & NUCLEAR ENGINEERING CO. INC. \\
\hline 41 & 364557 & 1164137 & 846.7 & 115.5 & 107.9 & 114.0 & 108.5 & $10-10-90$ & & U.S. ECOLOGY-W002 & U.S. ECOLOGY \\
\hline 42 & 364556 & 1164135 & 1456.0 & 123.1 & 110.9 & 123.1 & 112.5 & $02-06-87$ & & WELL MR3 & USGS \\
\hline 43 & 364554 & 1164855 & 987.5 & 97.8 & & & 74.5 & $01-11-87$ & & & USGS \\
\hline 44 & 364553 & 1164138 & 846.4 & & & & & $00-00-00$ & & WEST TRENCH 13 & \\
\hline 45 & 364553 & 1164138 & 846.4 & & & & & $00-00-00$ & & WEST TRENCH 14 & \\
\hline 46 & 364553 & 1164138 & 846.4 & & & & & $00-00-00$ & & WEST TRENCH 15 & \\
\hline 47 & 364553 & 1164138 & 846.4 & & & & & $00-00-00$ & & WEST TRENCH 16 & \\
\hline 48 & 364553 & 1164137 & 846.4 & & & & & $00-00-00$ & & EAST TRENCH 2 & \\
\hline 49 & 364553 & 1164137 & 846.4 & & & & & $00-00-00$ & & EAST TRENCH 3 & \\
\hline 50 & 364553 & 1164137 & 846.4 & & & & & $00-00-00$ & & EAST TRENCH 4 & \\
\hline 51 & 364553 & 1164137 & 846.4 & & & & & $00-00-00$ & & EAST TRENCH 5 & \\
\hline 52 & 364553 & 1164137 & 846.4 & & & & & $00-00-00$ & & EAST TRENCH 6 & \\
\hline 53 & 364553 & 1164137 & 846.4 & & & & & $00-00-00$ & & EAST TRENCH 7 & \\
\hline 54 & 364553 & 1164137 & 846.4 & & & & & $00-00-00$ & & EAST TRENCH 8 & \\
\hline 55 & 364553 & 1164138 & 846.4 & & & & & $00-00-00$ & & WEST TRENCH 9 & \\
\hline 56 & 364553 & 1164137 & 846.4 & & & & & $00-00-00$ & & EAST TRENCH 1 & \\
\hline 57 & 364553 & 1164138 & 846.4 & & & & & $00-00-00$ & & WEST TRENCH 10 & \\
\hline 58 & 364553 & 1164138 & 846.4 & & & & & $00-00-00$ & & WEST TRENCH 11 & \\
\hline 59 & 364553 & 1164138 & 846.4 & & & & & $00-00-00$ & & WEST TRENCH 12 & \\
\hline 60 & 364552 & 1164133 & 847.3 & 126.2 & 86.9 & 126.2 & 109.4 & $02-27-85$ & & & USGS \\
\hline
\end{tabular}




\begin{tabular}{|c|c|c|c|c|c|c|c|c|c|c|}
\hline $\begin{array}{l}\text { Site } \\
\text { No }\end{array}$ & Lat & Long & $\begin{array}{l}\text { Land } \\
\text { Elev }\end{array}$ & $\begin{array}{l}\text { Total } \\
\text { Depth }\end{array}$ & $\begin{array}{l}\text { Perfo } \\
\text { top }\end{array}$ & $\begin{array}{l}\text { ration } \\
\text { bottom }\end{array}$ & $\begin{array}{l}\text { Water } \\
\text { Level }\end{array}$ & $\begin{array}{l}\text { Measure } \\
\text { Date }\end{array}$ & $\begin{array}{l}\text { Note } \\
\text { O C }\end{array}$ & Site Name \\
\hline 61 & 364550 & 1164727 & 926.5 & 143.2 & & & 127.0 & $01-11-87$ & & \\
\hline 62 & 364514 & 1164829 & 981.4 & 115.8 & & & 108.1 & $01-01-87$ & & \\
\hline 63 & 364300 & 1163714 & 792.4 & 147.5 & & & & $01-11-87$ & D & \\
\hline 64 & 364247 & 1164423 & 829.0 & 579.1 & 585.2 & 588.2 & 92.4 & $01-11-87$ & & \\
\hline 65 & 364246 & 1164457 & 829.0 & 426.7 & & & 85.8 & $01-11-87$ & & \\
\hline 66 & 364243 & 1164324 & 822.9 & 566.9 & 524.2 & 566.9 & 92.8 & $01-11-87$ & & \\
\hline 67 & 364130 & 1164112 & 800.9 & 292.6 & 283.5 & 286.5 & 82.2 & $08-03-86$ & & NA-6 WELL BGMW-10 \\
\hline 68 & 364130 & 1164112 & 804.6 & 98.4 & 97.5 & 98.4 & 82.1 & $01-11-87$ & & \\
\hline 69 & 364105 & 1163026 & 831.1 & 140.2 & 97.5 & 140.2 & 103.6 & $06-30-92$ & & CIND-R-LITE WELL \\
\hline 70 & 364044 & 1163753 & 773.5 & 54.3 & & & & $00-00-00$ & & \\
\hline 71 & 363934 & 1162558 & 816.5 & 74.1 & & & & $00-00-00$ & & VH-2 \\
\hline 72 & 363907 & 1162357 & 819.9 & 143.6 & 31.7 & 143.6 & 101.5 & $05-20-61$ & & \\
\hline 73 & 363858 & 1162541 & 804.6 & 23.8 & & & & $11-11-53$ & D & \\
\hline 74 & 363842 & 1162353 & 811.3 & 163.1 & 109.4 & 163.1 & 111.2 & $01-03-64$ & & \\
\hline 75 & 363842 & 1162353 & 811.3 & 163.1 & 109.4 & 163.1 & 106.0 & $09-12-90$ & & \\
\hline 76 & 363840 & 1162340 & 810.7 & 120.4 & 102.1 & 120.4 & 105.2 & $05-03-52$ & & \\
\hline 77 & 363840 & 1162350 & 813.8 & 153.9 & 109.7 & 153.9 & 109.7 & $02-28-55$ & & \\
\hline 78 & 363838 & 1162345 & 811.3 & 154.5 & 115.8 & 154.5 & 109.7 & $03-08-55$ & & \\
\hline 79 & 363838 & 1162345 & 811.3 & 154.5 & 115.8 & 154.5 & 109.7 & $03-08-55$ & & \\
\hline 80 & 363838 & 1162341 & 811.3 & 109.7 & & & & $00-00-00$ & & \\
\hline 81 & 363835 & 1162340 & 809.8 & 150.9 & 120.4 & 150.9 & 111.2 & $04-30-72$ & & NDOT WELL \\
\hline 82 & 363825 & 1162433 & 804.3 & 228.6 & 109.7 & 236.8 & 88.4 & $02-20-64$ & & AIRPORT WELL \\
\hline 83 & 363825 & 1162632 & 795.5 & 173.7 & 115.8 & 173.7 & 90.1 & $01-15-87$ & & \\
\hline 84 & 363815 & 1161759 & 931.4 & 243.8 & 224.0 & 243.8 & 205.4 & $07-21-62$ & & TW- 5 \\
\hline 85 & 363750 & 1162000 & & & & & & $00-00-00$ & & \\
\hline 86 & 363749 & 1161302 & 1807.4 & 112.8 & 102.1 & & & $10-01-59$ & & \\
\hline 87 & 363744 & 1162637 & 783.9 & 151.8 & 151.8 & & 77.8 & $07-12-62$ & & \\
\hline 88 & 363740 & 1162639 & & & & & & $00-00-00$ & & \\
\hline 89 & 363715 & 1162445 & 853.4 & 32.0 & & & & $00-00-00$ & & \\
\hline 90 & 363709 & 1162646 & 775.7 & & & & 71.7 & $01-15-87$ & & \\
\hline
\end{tabular}

Owner

USGS

USGS

DALE, W.

USGS

USGS

USGS

USGS

USGS

CIND-R-LITE LAS VEGAS,NV

WASHBURN, RICHARD

BOSSINGHAM, BEN

WASHBURN, RICHARD

COBB, FRED

KAY, JOHN

PEREIDRA, LOUISE

WHELLOCK, BOB

JOE RICHARDS

PEREIDRA, LOUISE

NEVADA HIGHWAY DEPARTMENT

DOING, WARREN

SHAW, JAMES $H$.

U.S. GOVT

JOE FALLIN

WASHBURN, RICHARD

NYE COUNTY LAND CO.

WASHBURN, RICHARD 


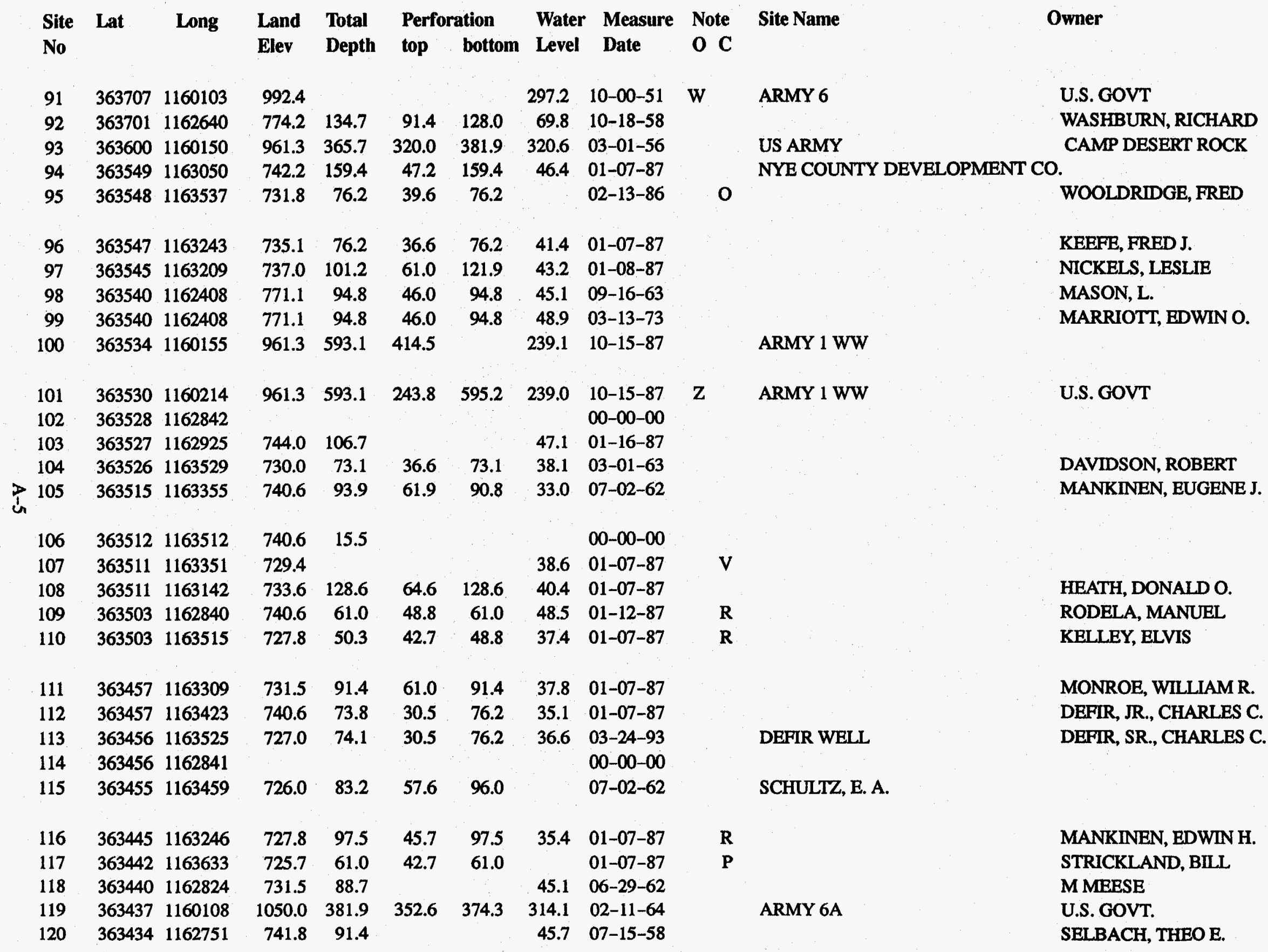




\begin{tabular}{|c|c|c|c|c|c|c|c|c|c|c|c|}
\hline $\begin{array}{l}\text { Site } \\
\text { No }\end{array}$ & Lat & Long & $\begin{array}{l}\text { Land } \\
\text { Elev }\end{array}$ & $\begin{array}{l}\text { Total } \\
\text { Depth }\end{array}$ & $\begin{array}{l}\text { Perfo } \\
\text { top }\end{array}$ & $\begin{array}{l}\text { ation } \\
\text { bottom }\end{array}$ & $\begin{array}{l}\text { Water } \\
\text { Level }\end{array}$ & $\begin{array}{l}\text { Measure } \\
\text { Date }\end{array}$ & $\begin{array}{l}\text { Note } \\
\text { O C }\end{array}$ & Site Name & Owner \\
\hline 121 & 363432 & 1163442 & 723.3 & 105.8 & 32.0 & 105.8 & 33.2 & $01-20-84$ & & & CALDWELL, C. L. \\
\hline 122 & 363430 & 1162647 & 745.2 & 51.2 & & & 55.7 & $01-13-87$ & & & \\
\hline 123 & 363430 & 1162452 & 762.0 & 136.5 & & & 53.0 & $06-29-62$ & & & \\
\hline 124 & 363429 & 1163159 & 729.0 & 91.4 & 53.6 & 91.4 & 36.9 & $01-07-87$ & & & PIERCE, JAMES K. \\
\hline 125 & 363429 & 1162334 & 755.3 & & & & 39.6 & $03-16-87$ & & & COOK, LEWIS C. \\
\hline 126 & 363428 & 1163215 & 740.6 & 87.8 & 39.6 & 92.0 & 34.1 & $07-04-62$ & & & PIERCE, JAMES K. \\
\hline 127 & 363428 & 1162812 & 738.2 & & & & 47.8 & $01-12-87$ & & AMARGOSA WATER & NYE COUNTY \\
\hline 128 & 363428 & 1162347 & 755.2 & 82.0 & 44.8 & 82.0 & 43.9 & $12-20-61$ & & COOKS EAST WELL & COOK, LEWIS, C. \\
\hline 129 & 363428 & 1162403 & 754.2 & 95.1 & 54.3 & 91.1 & 33.9 & $04-09-91$ & & COOKS WEST WELL & COOK, LEWIS C. \\
\hline 130 & 363425 & 1162350 & & & & & & $00-00-00$ & & & \\
\hline 131 & 363425 & 1163320 & & & & & & $00-00-00$ & & FOX WAS KIRKER WELL & \\
\hline 132 & 363422 & 1162534 & 755.9 & 61.0 & & & 42.7 & $07-31-62$ & & & COOK, L. \\
\hline 133 & 363418 & 1162742 & & & & & & $00-00-00$ & & & \\
\hline 134 & 363417 & 1162730 & 740.6 & 57.9 & & & 52.4 & $01-20-84$ & & & NYE COUNTY LAND \\
\hline 135 & 363415 & 1160926 & 846.4 & & & & & $00-00-00$ & & & \\
\hline 136 & 363411 & 1162738 & 739.1 & 97.5 & 85.3 & 97.5 & & $00-00-00$ & & AMARGOSA ELEMENTARY & NYE COUNTY SCHOOL DISTRICT \\
\hline 137 & 363411 & 1162729 & 739.1 & 91.4 & 61.0 & 91.4 & 50.3 & $11-19-80$ & & AMARGOSA TOWN COMPLEX & NYE COUNTY \\
\hline 138 & 363410 & 1162611 & 743.7 & 204.2 & 64.6 & 204.2 & 52.5 & $01-15-87$ & & & NYE COUNTY DEVELOPMENT CO. \\
\hline 139 & 363410 & 1162400 & 749.8 & 53.6 & 44.8 & 87.2 & 26.2 & $03-16-87$ & & & COOK, LEWIS C. \\
\hline 140 & 363410 & 1162403 & 748.6 & 61.0 & 50.3 & 57.9 & 34.6 & $03-16-87$ & & & COOK, L. C. \\
\hline 141 & 363410 & 1162735 & & & & & & $00-00-00$ & & & \\
\hline 142 & 363407 & 1162733 & 737.9 & 91.4 & 61.0 & 91.4 & 36.6 & $12-10-88$ & & AMARGOSA VALLEY WATER & NYE COUNTY \\
\hline 143 & 363405 & 1163240 & & & & & & $00-00-00$ & & & \\
\hline 144 & 363405 & 1163551 & 726.9 & 125.0 & 43.9 & 125.0 & & $00-00-00$ & & & BOWLINE, ARNOLD \\
\hline 145 & 363405 & 1163345 & 723.9 & 76.2 & & & 27.4 & $08-15-58$ & & & SELBACH, EARL N. \\
\hline 146 & 363404 & 1162504 & 746.7 & 88.4 & 15.5 & 88.4 & 48.2 & $06-29-62$ & & & JOHNS, WILLARD \\
\hline 147 & 363404 & 1163239 & 724.2 & 45.7 & & & 31.5 & $02-26-74$ & & & MANKINEN, E. H. \\
\hline 148 & 363404 & 1163312 & 724.2 & 92.0 & 45.1 & 89.0 & 31.8 & $01-08-87$ & & & DANSBY, LEWIS N. \\
\hline 149 & 363402 & 1163442 & 721.4 & & & & 31.9 & $01-07-87$ & & & \\
\hline 150 & 363401 & 1162617 & 740.6 & 118.9 & 45.7 & 128.0 & 49.8 & $01-20-84$ & & & JOHNS, WILMA A. \\
\hline
\end{tabular}




\begin{tabular}{|c|c|c|c|c|c|c|c|c|c|c|c|}
\hline $\begin{array}{l}\text { Site } \\
\text { No }\end{array}$ & Lat & Long & $\begin{array}{l}\text { Land } \\
\text { Elev }\end{array}$ & $\begin{array}{l}\text { Total } \\
\text { Depth }\end{array}$ & $\begin{array}{l}\text { Perfor } \\
\text { top }\end{array}$ & $\begin{array}{l}\text { ation } \\
\text { bottom }\end{array}$ & $\begin{array}{l}\text { Water } \\
\text { Level }\end{array}$ & $\begin{array}{l}\text { Measure } \\
\text { Date }\end{array}$ & $\begin{array}{l}\text { Note } \\
\text { O C }\end{array}$ & Site Name & Owner \\
\hline 151 & 363401 & 1163510 & 722.0 & 85.3 & 36.6 & 85.3 & 33.2 & $01-20-84$ & & & OVERHALSER, J. H. \\
\hline 152 & 363400 & 1163206 & 725.7 & 89.9 & 18.3 & 89.9 & 34.0 & $01-08-87$ & & & GALLAGHER, TOM \\
\hline 153 & 363356 & 1162945 & 726.9 & 128.0 & 42.7 & 128.0 & 35.4 & $06-28-62$ & & & HANKS, WILLIAM T. \\
\hline 154 & 363355 & 1162549 & 742.2 & 139.3 & 61.6 & 139.3 & 50.0 & $01-15-87$ & & & JOHNS, WILLARD \\
\hline 155 & 363353 & 1163033 & 727.2 & 76.2 & & & 37.2 & $01-08-87$ & & & PHILLIPS, TOM \\
\hline 156 & 363352 & 1163628 & 720.2 & 110.0 & 42.7 & 115.8 & 35.8 & $01-20-84$ & & & BELL, JOHN S. \\
\hline 157 & 363348 & 1162549 & 743.7 & 118.9 & 45.7 & 118.9 & 51.1 & $03-21-86$ & & & JOHNS, WILLARD \\
\hline 158 & 363338 & 1163031 & 722.3 & 91.4 & 30.5 & 88.4 & & $01-08-87$ & D & & PERRY, P. J. \\
\hline 159 & 363338 & 1163031 & 722.3 & 91.4 & 30.5 & 88.4 & 28.0 & $11-26-58$ & & & SCOTT, ARTHUR E. \\
\hline 160 & 363336 & 1162930 & 726.3 & 75.6 & & & 35.3 & $06-28-62$ & & & \\
\hline 161 & 363333 & 1160642 & 858.9 & & & & 138.5 & $04-29-64$ & W & WELLS - STEWART & WELLS STEW \\
\hline 162 & 363332 & 1163250 & 720.5 & 61.0 & 27.4 & 61.0 & 29.4 & $01-08-87$ & $\mathbf{R}$ & & SPEARS, I. C. \\
\hline 163 & 363330 & 1160700 & & 243.8 & & & & $00-00-00$ & & PT OF ROCKS HWY-STEWART & \\
\hline 164 & 363323 & 1162944 & & & & & & $00-00-00$ & & & \\
\hline 165 & 363320 & 1162809 & & & & & & $00-00-00$ & & ALBITRE WELL WAS THIEDE & \\
\hline 166 & 363316 & 1163624 & 719.3 & 53.3 & 30.5 & 53.3 & 30.1 & $01-07-87$ & $\mathbf{R}$ & & THARP, ELDON \\
\hline 167 & 363315 & 1163031 & 721.7 & & & & 28.8 & $05-24-56$ & & & DE LEE, MORRIS \\
\hline 168 & 363315 & 1163031 & 721.7 & & & & 35.1 & $01-19-84$ & & & RECORDS, MARY \\
\hline 169 & 363313 & 1163025 & & & & & & $00-00-00$ & & & \\
\hline 170 & 363311 & 1163139 & 718.7 & 100.6 & 24.4 & 100.6 & 33.1 & $01-10-87$ & & & GILLESPIE, HAROLD \\
\hline 171 & 363310 & 1162940 & 724.3 & 106.1 & & & 31.7 & $08-27-53$ & & USBLM WELL & USBLM \\
\hline 172 & 363309 & 1162821 & 726.6 & 91.4 & 33.5 & 91.4 & 30.5 & $10-31-59$ & & & TRAVIS, EARL \\
\hline 173 & 363309 & 1162821 & 726.6 & 91.4 & 33.5 & 91.4 & 39.2 & $01-13-87$ & & & TRAVIS, MAX/EARL, JACK/CLEMENT, PAUI \\
\hline 174 & 363309 & 1162650 & 730.0 & 106.7 & 45.7 & 106.7 & 42.8 & $01-13-87$ & & & DALTON, RALPH C. \\
\hline 175 & 363303 & 1162929 & 723.6 & 146.3 & 39.6 & 146.3 & & $12-11-63$ & D & & MEETER, LEO \\
\hline 176 & 363303 & 1163001 & 722.6 & 221.0 & 30.5 & 221.0 & 2.1 & $02-15-55$ & & & MEETER, L. \\
\hline 177 & 363303 & 1162513 & 732.4 & 152.4 & 36.6 & 152.4 & 32.8 & $01-13-87$ & & & DALTON, RALPH C. \\
\hline 178 & 363303 & 1163001 & 722.6 & 221.0 & 30.5 & 221.0 & 2.1 & $02-15-55$ & & & DELEE, MORRIS \\
\hline 179 & 363303 & 1163001 & 722.6 & 221.0 & 30.5 & 221.0 & 34.8 & $01-10-87$ & & & RECORDS, HENRY H. \\
\hline 180 & 363303 & 1162929 & 723.6 & 146.3 & 39.6 & 146.3 & 24.4 & $01-25-55$ & & & RECORDS, R. D. \\
\hline
\end{tabular}




\begin{tabular}{|c|c|c|c|c|c|c|c|c|c|c|c|}
\hline $\begin{array}{l}\text { Site } \\
\text { No }\end{array}$ & Lat & Long & $\begin{array}{l}\text { Land } \\
\text { Elev }\end{array}$ & $\begin{array}{l}\text { Total } \\
\text { Depth }\end{array}$ & $\begin{array}{l}\text { Perfor } \\
\text { top }\end{array}$ & $\begin{array}{l}\text { ation } \\
\text { bottom }\end{array}$ & $\begin{array}{l}\text { Water } \\
\text { Level }\end{array}$ & $\begin{array}{l}\text { Measure } \\
\text { Date }\end{array}$ & $\begin{array}{l}\text { Note } \\
\text { O C }\end{array}$ & Site Name & Owner \\
\hline 181 & 363302 & 1163218 & 718.7 & 100.6 & 30.5 & 100.6 & 28.6 & $03-23-60$ & & & GILLESPIE, HAROLD \\
\hline 182 & 363252 & 1163230 & & & & & & $00-00-00$ & & RANCHO AMARGOSA WELL & \\
\hline 183 & 363249 & 1162919 & & & & & & $00-00-00$ & & & \\
\hline 184 & 363248 & 1163028 & 718.4 & & & & 32.6 & $01-10-87$ & & & DE LEE, MORRIS \\
\hline 185 & 363247 & 1163129 & 715.6 & 91.4 & 27.4 & 91.4 & 32.2 & $01-10-87$ & & & GILLESPIE, HAROLD \\
\hline 186 & 363246 & 1162922 & 720.1 & 93.6 & & & 30.0 & $07-09-62$ & & & L MEETER \\
\hline 187 & 363243 & 1163541 & 714.4 & & & & 26.7 & $01-08-87$ & & & HOLTZ, CHARLES \\
\hline 188 & 363242 & 1162839 & 721.1 & 125.0 & 47.5 & 125.0 & 33.5 & $03-10-59$ & & & EASTABROOK, E. R. \\
\hline 189 & 363242 & 1163019 & 718.7 & 91.4 & 30.5 & 91.4 & 32.1 & $01-10-87$ & & & RECORDS, RUBY D. \\
\hline 190 & 363239 & 1163109 & 715.3 & 88.1 & & & 25.6 & $07-09-62$ & & & RECORDS, $\mathbf{R}$ \\
\hline 191 & 363239 & 1163144 & 746.7 & 153.3 & 82.3 & 153.3 & 23.7 & $07-07-62$ & & & GILLESPIE, VELEPIA \\
\hline 192 & 363237 & 1163033 & 718.4 & 128.3 & 42.7 & 128.3 & 28.2 & $12-02-60$ & & & MEETER, LEO J. \\
\hline 193 & 363237 & 1162929 & 720.2 & 91.4 & 36.6 & 93.6 & 24.4 & $12-23-54$ & & & RECORDS, H. H. \\
\hline 194 & 363237 & 1162929 & 720.2 & 91.4 & 36.6 & 93.6 & 24.4 & $12-23-54$ & & & DAVIS \\
\hline 195 & 363237 & 1162929 & 720.2 & 91.4 & 36.6 & 93.6 & 24.4 & $12-23-54$ & & & DELEE, MORRIS H. \\
\hline 196 & 363237 & 1162929 & 720.2 & 91.4 & 36.6 & 93.6 & 32.7 & $01-10-87$ & & & MEETER, LEO J. \\
\hline 197 & 363236 & 1162631 & 722.3 & 112.2 & 37.2 & 112.2 & 34.4 & $07-07-61$ & & & CYPERT, KEN \\
\hline 198 & 363236 & 1162631 & 722.3 & 112.2 & 37.2 & 112.2 & 36.9 & $02-12-86$ & & & PINKERTON, CHARLES W. \\
\hline 199 & 363234 & 1163217 & 713.5 & 147.8 & 51.8 & 147.8 & 22.9 & $07-07-62$ & & & GILLESPIE, HAROLD \\
\hline 200 & 363230 & 1162605 & 723.3 & & & & 36.8 & $01-13-87$ & & & BRIGHT, ROGER \\
\hline 201 & 363223 & 1163459 & 713.4 & 80.5 & 36.3 & 77.7 & 19.0 & $07-04-62$ & & & DOWNEY, JOHN W. \\
\hline 202 & 363219 & 1162958 & 715.6 & 90.2 & 57.9 & 81.7 & 27.4 & $04-11-66$ & & & DAVIS, KENNETH R. \\
\hline 203 & 363219 & 1162958 & 715.6 & 90.2 & 57.9 & 81.7 & 27.4 & $04-11-66$ & & & DELEE \\
\hline 204 & 363219 & 1162958 & 715.6 & 90.2 & 57.9 & 81.7 & & $01-14-87$ & 0 & & BETTLES, G. \\
\hline 205 & 363219 & 1163024 & & & & & & $00-00-00$ & & & \\
\hline 206 & 363218 & 1163134 & 712.0 & 42.1 & & & 30.6 & $01-19-84$ & & & G BETTLES \\
\hline 207 & 363217 & 1162658 & 721.7 & 76.2 & 30.5 & 74.7 & 36.1 & $01-13-87$ & & & CLAIRE, BUDDINGTON R. \\
\hline 208 & 363217 & 1162718 & 721.7 & 91.4 & 36.6 & 91.4 & 35.4 & $01-13-87$ & & & WICHNER, MILTON \\
\hline 209 & 363214 & 1162848 & 717.8 & 61.0 & 53.3 & 61.0 & 31.7 & $01-14-87$ & & & BROCKETT, PETE \\
\hline 210 & 363214 & 1161335 & 733.0 & 311.5 & 211.8 & 228.6 & 13.5 & $10-18-66$ & & AMARGOSA TRACER NO. 1 & U.S. GOVT \\
\hline
\end{tabular}




\begin{tabular}{|c|c|c|c|c|c|c|c|c|c|c|c|}
\hline $\begin{array}{l}\text { Site } \\
\text { No }\end{array}$ & Lat & Long & $\begin{array}{l}\text { Land } \\
\text { Elev }\end{array}$ & $\begin{array}{l}\text { Total } \\
\text { Depth }\end{array}$ & $\begin{array}{l}\text { Perf } \\
\text { top }\end{array}$ & $\begin{array}{l}\text { ation } \\
\text { bottom }\end{array}$ & $\begin{array}{l}\text { Water } \\
\text { Level }\end{array}$ & $\begin{array}{l}\text { Measure } \\
\text { Date }\end{array}$ & $\begin{array}{l}\text { Note } \\
\text { O C }\end{array}$ & Site Name & Owner \\
\hline 211 & 363213 & 1161339 & 731.5 & 249.3 & & & 12.0 & $03-15-87$ & & & USGS \\
\hline 212 & 363213 & 1161340 & 733.0 & 188.7 & & & 13.3 & $03-15-87$ & & & USGS \\
\hline 213 & 363213 & 1161337 & 733.0 & 202.4 & & & 13.5 & $03-15-87$ & & & USGS \\
\hline 214 & 363213 & 1161338 & 732.2 & 205.7 & 189.0 & 205.7 & 12.5 & $11-21-66$ & & TRACER WELL 3 & USGS \\
\hline 215 & 363211 & 1163032 & 714.4 & 50.0 & 18.3 & 45.7 & 25.6 & $10-02-21$ & & & LEALAND LAND \& WATER \\
\hline 216 & 363211 & 1161339 & & & & & & $00-00-00$ & & & \\
\hline 217 & 363211 & 1163032 & 714.4 & 50.0 & 18.3 & 45.7 & 25.6 & $10-02-21$ & & & BETTLES, G. \\
\hline 218 & 363211 & 1163032 & 714.4 & 50.0 & 18.3 & 45.7 & 25.6 & $10-02-21$ & & & DE LEE, MORRIS \\
\hline 219 & 363211 & 1163032 & 714.4 & 50.0 & 18.3 & 45.7 & 30.9 & $01-08-87$ & $\mathbf{R}$ & & T \& T RANCH \\
\hline 220 & 363211 & 1162946 & 715.6 & 54.9 & & & & $12-11-63$ & D & & LEALAND LAND \& WATER \\
\hline 221 & 363211 & 1161339 & & & & & & $00-00-00$ & & AMARGOSA TR HOLE 2 SUMP & \\
\hline 222 & 363211 & 1161339 & & & & & & $00-00-00$ & & AMARGOSA TR HOLE 2 & \\
\hline 223 & 363210 & 1163138 & 712.6 & 61.0 & & & 23.7 & $01-01-52$ & & & DAVIS \\
\hline 224 & 363210 & 1163138 & 712.6 & 61.0 & & & 30.0 & $01-13-87$ & & & LEALAND LAND \& WATER \\
\hline 225 & 363209 & 1163140 & 701.0 & 60.3 & & & 23.3 & $02-24-75$ & & & GORDON BETTLS \\
\hline 226 & 363200 & 1163233 & 710.1 & 61.0 & 19.8 & 61.0 & 16.5 & $03-12-56$ & & & DELPH, M. M. \\
\hline 227 & 363155 & 1163233 & 707.7 & 71.9 & 32.3 & 71.9 & 18.3 & $01-27-66$ & & & BARR, MILDRED A. \\
\hline 228 & 363153 & 1162216 & 723.6 & 30.5 & & & 30.6 & $03-15-87$ & & IMV BENTONITE MINE WELL & \\
\hline 229 & 363150 & 1163116 & 709.2 & 15.5 & & & 20.5 & $07-09-62$ & & & BETTLES, G. \\
\hline 230 & 363148 & 1162510 & 716.2 & 59.4 & 41.1 & 59.4 & 40.7 & $01-15-87$ & & & WILBURN, M. H. /GRUBB, MARJORIE \\
\hline 231 & 363148 & 1163238 & 708.5 & $\mathbf{5 7 . 3}$ & & & 22.5 & $01-10-87$ & & & DELPH, M. M. \\
\hline 232 & 363146 & 1162720 & 717.5 & 91.4 & 35.7 & 91.4 & 29.5 & $06-26-62$ & & & WICHNER, MILTON \\
\hline 233 & 363146 & 1162527 & 716.5 & 91.4 & & & 25.1 & $06-26-62$ & & & BILSBOROUGH, DONALD L. \\
\hline 234 & 363145 & 1163106 & 710.1 & 61.0 & & & & $08-16-78$ & D & & LEALAND LAND \& WATER \\
\hline 235 & 363138 & 1161416 & 722.5 & 8.8 & & & 21.3 & $01-01-07$ & $\mathrm{D}$ & & MILLER \\
\hline 236 & 363138 & 1162505 & & 91.4 & 42.7 & 91.4 & 32.6 & $06-26-62$ & & & RECORDS, MORNA \\
\hline 237 & 363138 & 1162505 & & 61.0 & 45.7 & 61.0 & 38.1 & $06-25-61$ & & & MARTIN, JACK A. \\
\hline 238 & 363134 & 1162920 & 710.1 & 55.2 & 31.1 & 55.2 & 25.5 & $01-14-87$ & & & OWENS, JM \\
\hline 239 & 363133 & 1162501 & 713.2 & 48.8 & & & 37.9 & $01-12-87$ & & & GOOD \\
\hline 240 & 363132 & 1162400 & & & & & & $00-00-00$ & & & \\
\hline
\end{tabular}




\begin{tabular}{|c|c|c|c|c|c|c|c|c|c|c|c|}
\hline $\begin{array}{l}\text { Site } \\
\text { No }\end{array}$ & Lat & Long & $\begin{array}{l}\text { Land } \\
\text { Elev }\end{array}$ & $\begin{array}{l}\text { Total } \\
\text { Depth }\end{array}$ & $\begin{array}{l}\text { Perfol } \\
\text { top }\end{array}$ & $\begin{array}{l}\text { ation } \\
\text { bottom }\end{array}$ & $\begin{array}{l}\text { Water } \\
\text { Level }\end{array}$ & $\begin{array}{l}\text { Measure } \\
\text { Date }\end{array}$ & $\begin{array}{l}\text { Note } \\
\text { O C }\end{array}$ & Site Name & Owner \\
\hline 241 & 363132 & 1162753 & 715.9 & 61.0 & 32.6 & 61.0 & 33.8 & $01-18-84$ & & & MASON, EVA \\
\hline 242 & 363132 & 1162857 & 712.6 & 25.0 & & & 24.7 & $06-26-62$ & & & GAMETT, LOU W. \\
\hline 243 & 363132 & 1162852 & 710.8 & & & & 25.4 & $01-14-87$ & & & \\
\hline 244 & 363129 & 1162417 & 713.2 & 61.0 & 30.5 & 61.0 & 36.1 & $03-18-87$ & & & NICKELL, JERALD W. \\
\hline 245 & 363129 & 1162425 & 712.6 & 61.0 & 45.7 & 61.0 & 37.3 & $01-15-87$ & & & MILLS, JOHNNIE \\
\hline 246 & 363129 & 1162529 & 713.2 & 61.0 & 53.3 & 61.0 & 36.8 & $01-12-87$ & & & LEIS, L. \\
\hline 247 & 363128 & 1163024 & & & & & & $00-00-00$ & & & \\
\hline 248 & 363128 & 1162531 & 713.5 & 68.0 & 30.5 & 68.0 & 25.9 & $03-15-59$ & & & MCCOY, ED \\
\hline 249 & 363123 & 1163000 & 709.0 & 46.6 & & & 21.2 & $06-26-62$ & & & G BETTLES \\
\hline 250 & 363121 & 1161123 & 745.0 & 45.7 & 29.0 & 45.7 & 30.3 & $08-30-62$ & & & DOOLEY, WILLIAM J. \\
\hline 251 & 363120 & 1163000 & 708.6 & 49.4 & & & & $03-19-92$ & 0 & BETTLES & BETTLES, GORDON W. \\
\hline 252 & 363117 & 1163036 & 708.9 & 49.1 & 16.8 & 61.0 & 25.3 & $01-10-87$ & $\mathbf{V}$ & & BETTLES, BILLIE \\
\hline 253 & 363117 & 1163036 & 708.9 & 49.1 & 16.8 & 61.0 & 14.0 & $02-15-56$ & & & BOYD, DAVID C. \\
\hline 254 & 363115 & 1162420 & 740.6 & & & & 36.7 & $01-18-84$ & & & BRADLEY \\
\hline 255 & 363111 & 1162756 & 712.0 & 91.4 & 30.5 & 91.4 & 24.5 & $06-26-62$ & & & HODGES, MITCHELL \\
\hline 256 & 363111 & 1162902 & 709.5 & 26.8 & & & & $06-26-62$ & $\mathbf{D}$ & & HOUSSELL, J. KELL \\
\hline 257 & 363109 & 1162526 & 708.0 & 61.0 & 33.5 & 61.0 & 30.2 & $06-27-62$ & & & BERRY, N. O. \\
\hline 258 & 363100 & 1162814 & 707.1 & 77.1 & 28.6 & 75.6 & 24.7 & $01-14-87$ & & & STEPHENS, MELVIN M. \\
\hline 259 & 363058 & 1162705 & 707.1 & 113.4 & 48.8 & 113.4 & 29.3 & $01-16-87$ & & & HONIG, MARILYN \\
\hline 260 & 363058 & 1163128 & 703.7 & 91.4 & 21.3 & 91.4 & 19.9 & $01-10-87$ & & & BARR, CHARLES M. \\
\hline 261 & 363058 & 1162857 & 706.2 & 24.4 & & & 19.4 & $06-26-62$ & & & HOUSSELL, J. KELL \\
\hline 262 & 363054 & 1162704 & 706.5 & 91.4 & 32.0 & 91.4 & 27.7 & $07-06-62$ & & & HONIG, MARILYN \\
\hline 263 & 363053 & 1162720 & 706.5 & 92.0 & 32.3 & 89.0 & 25.0 & $04-16-68$ & & & NYE COUNTY DEVELOPMENT CO. \\
\hline 264 & 363053 & 1162710 & 704.7 & & & & 27.7 & $01-14-87$ & & & \\
\hline 265 & 363050 & 1161050 & 732.4 & 41.1 & 18.3 & 41.1 & 17.7 & $02-01-61$ & & & WHITE, WILLIAM R. \\
\hline 266 & 363044 & 11627327 & 40.6 & 97.8 & & & 25.5 & $06-20-62$ & & & HONIG, P. \\
\hline 267 & 363044 & 1163050 & 701.9 & 124.0 & 50.3 & 124.0 & 16.5 & $01-14-69$ & & & WATSON, HELEN \\
\hline 268 & 363039 & 1163035 & 702.8 & & & & 21.1 & $01-12-87$ & & & BETTLES, GORDON W. \\
\hline 269 & 363031 & 1161047 & 732.4 & 41.1 & 18.3 & 41.1 & 17.7 & $02-01-60$ & & WHITE WELL & WHITE \\
\hline 270 & 363030 & 1162800 & 706.2 & & & & 20.7 & $01-20-56$ & & & MOORE, JR., WILLIAM \\
\hline
\end{tabular}




\begin{tabular}{|c|c|c|c|c|c|c|c|c|c|c|c|}
\hline $\begin{array}{l}\text { Site } \\
\text { No }\end{array}$ & Lat & Long & $\begin{array}{l}\text { Land } \\
\text { Elev }\end{array}$ & $\begin{array}{l}\text { Total } \\
\text { Depth }\end{array}$ & $\begin{array}{l}\text { Perfo } \\
\text { top }\end{array}$ & $\begin{array}{l}\text { ation } \\
\text { bottom }\end{array}$ & $\begin{array}{l}\text { Water } \\
\text { Level }\end{array}$ & $\begin{array}{l}\text { Measure } \\
\text { Date }\end{array}$ & $\begin{array}{l}\text { Note } \\
\text { O C }\end{array}$ & Site Name & Owner \\
\hline 271 & 363029 & 1162804 & 706.2 & 27.7 & & & 25.7 & $06-26-62$ & & & MOORE, JR., WILLIAM \\
\hline 272 & 363029 & 1162924 & 704.1 & 20.7 & & & 18.0 & $12-22-55$ & & & CLEVELAND, ELDON L. \\
\hline 273 & 363028 & 1162702 & 702.2 & 106.7 & 48.8 & 106.7 & 26.8 & $03-21-86$ & & & NYE COUNTY DEVELOPMENT CO. \\
\hline 274 & 363028 & 1163025 & & & & & & $00-00-00$ & & & \\
\hline 275 & 363028 & 1162530 & 701.3 & 91.4 & 27.4 & 61.0 & 24.8 & $06-26-62$ & & & BERRY, N. O. \\
\hline 276 & 363027 & 1162700 & 702.2 & 179.8 & 67.1 & 179.8 & 25.0 & $10-17-62$ & & & NYE COUNTY DEVELOPMENT CO. \\
\hline 277 & 363027 & 1162952 & 702.5 & 47.2 & 20.1 & 47.2 & 20.1 & $01-12-87$ & & & TYNAN, J. C. \\
\hline 278 & 363026 & 1162756 & 705.6 & 80.5 & 50.6 & 77.4 & 28.2 & $01-12-87$ & & & STRAUSS, HERMAN \\
\hline 279 & 363026 & 1161604 & 710.1 & & & & 24.5 & $03-09-72$ & & & SPRING MEADOWS INC. \\
\hline 280 & 363026 & 1161604 & 710.1 & & & & 30.7 & $03-09-72$ & & & \\
\hline 281 & 363020 & 1162900 & 701.0 & 24.4 & & & 19.5 & $01-17-56$ & & & MOORE, PATRICIA \\
\hline 282 & 363017 & 1162530 & 702.5 & 61.0 & 36.6 & 61.0 & 18.3 & $05-07-57$ & & & BERRY, HELEN S. \\
\hline 283 & 363009 & 1163027 & 702.5 & 34.1 & 22.2 & 34.1 & 14.6 & $11-17-57$ & & HALLOWELL WELL & HALLOWELL, DAVID \\
\hline 284 & 363005 & 1162910 & 700.4 & & & & 17.4 & $01-10-56$ & & & CLEVELAND, ELDON L. \\
\hline 285 & 363002 & 1163107 & 694.9 & 65.2 & & & 13.3 & 02-14-55 & & & ERICKSON, JULIUS \\
\hline 286 & 363002 & 1163107 & 694.9 & 65.2 & & & 13.6 & $03-13-73$ & & & HOYLE, W. G. \\
\hline 287 & 363002 & 1163048 & 698.0 & & & & 17.4 & $01-12-87$ & & & \\
\hline 288 & 363001 & 1162541 & 698.6 & 58.2 & & & 21.6 & $06-26-62$ & & & \\
\hline 289 & 362958 & 1163022 & 701.0 & 61.9 & 18.3 & 61.6 & 17.0 & $01-19-84$ & & & BETTLES, ALVIN E. \\
\hline 290 & 362957 & 1162549 & 698.0 & & & & 24.2 & $01-12-87$ & & & MARTINSON, JOSE \\
\hline 291 & 362947 & 1162520 & 694.0 & 64.0 & 32.0 & 64.0 & 21.0 & $01-12-87$ & & MOUNTAIN VIEW HOMES & DOUGLAS COUNTY \\
\hline 292 & 362946 & 1163023 & 698.9 & 60.0 & 9.1 & 60.0 & 12.2 & $04-24-60$ & & & BETTLES, ALVIN \\
\hline 293 & 362946 & 1162724 & 698.0 & & & & 21.1 & $09-14-62$ & & & NYE COUNTY DEVELOPMENT CO. \\
\hline 294 & 362946 & 1163023 & 701.0 & 32.0 & 13.1 & 32.0 & 12.2 & $06-20-60$ & & & BETTLES, ALVIN \\
\hline 295 & 362940 & 1162658 & & & & & & $00-00-00$ & & & \\
\hline 296 & 362939 & 1162654 & 688.8 & & & & 21.1 & $01-12-87$ & & & \\
\hline 297 & 362938 & 1163001 & & & & & 11.9 & $12-02-55$ & & & DAVIS, $\mathrm{H}$. \\
\hline 298 & 362938 & 1163041 & 698.0 & & & & & $00-00-00$ & & AMARGOSA WATER CO & \\
\hline 299 & 362938 & 1162700 & 695.2 & 91.4 & 30.5 & 91.4 & 23.2 & $01-12-87$ & & & AMARGOSA FARMS \\
\hline 300 & 362938 & 1162931 & 701.0 & 64.0 & 9.1 & 64.0 & 16.7 & $06-25-62$ & & & DAVIS, T. \\
\hline
\end{tabular}




\begin{tabular}{|c|c|c|c|c|c|c|c|c|c|c|c|}
\hline $\begin{array}{l}\text { Site } \\
\text { No }\end{array}$ & Lat & Long & $\begin{array}{l}\text { Land } \\
\text { Elev }\end{array}$ & $\begin{array}{l}\text { Total } \\
\text { Depth }\end{array}$ & $\begin{array}{l}\text { Perfor } \\
\text { top }\end{array}$ & $\begin{array}{l}\text { ation } \\
\text { bottom }\end{array}$ & $\begin{array}{l}\text { Water } \\
\text { Level }\end{array}$ & $\begin{array}{l}\text { Measure } \\
\text { Date }\end{array}$ & $\begin{array}{l}\text { Note } \\
\text { O C }\end{array}$ & Site Name & Owner \\
\hline 301 & 362937 & 1162533 & 693.3 & 48.8 & 13.7 & 48.8 & 20.1 & $01-12-87$ & & & BETTLES, GORDON \\
\hline 302 & 362936 & 1162812 & 696.3 & 18.0 & & & 17.6 & $05-11-62$ & & & CLEVELAND, ANETTA L. \\
\hline 303 & 362936 & 1162515 & & 91.4 & 24.4 & 91.4 & 18.0 & $03-20-62$ & & & SIEGEL, LEE \\
\hline 304 & 362935 & 1163025 & 701.0 & 25.9 & & & 12.8 & $12-06-55$ & & & BETTLES, ALEX E. \\
\hline 305 & 362934 & 1162959 & 701.0 & 152.4 & & & 12.4 & $06-25-62$ & & & H DAVIS \\
\hline 306 & 362932 & 1162741 & 696.6 & 146.3 & 45.7 & 146.3 & 47.4 & $06-20-62$ & & & GEERS, RAYMOND T. \\
\hline 307 & 362932 & 1162741 & 696.6 & 146.3 & 45.7 & 146.3 & 24.4 & $07-14-59$ & & & WALL, STEPHEN E. \\
\hline 308 & 362930 & 1162959 & 698.0 & & & & 21.8 & $01-12-87$ & & & \\
\hline 309 & 362929 & 1160857 & 729.7 & 65.5 & & & 11.2 & $02-04-60$ & & CHERRY PATCH WELL & CLARK, HERSHEL \& ETAL \\
\hline 310 & 362927 & 1161026 & 725.4 & 38.1 & & & 10.7 & $10-12-84$ & & & MORRELL, JEANNINE \\
\hline 311 & 362921 & 1163025 & 696.1 & 25.9 & & & & $00-00-00$ & & & \\
\hline 312 & 362920 & 1163110 & & & & & & $00-00-00$ & & & \\
\hline 313 & 362917 & 1163047 & 698.0 & & & & 14.5 & $01-12-87$ & $\mathbf{R}$ & & \\
\hline 314 & 362911 & 1162949 & 701.0 & & & & 13.4 & $12-03-55$ & & & DAVIS, $\mathrm{H}$. \\
\hline 315 & 362911 & 1162928 & 701.0 & 121.9 & & & 17.5 & $06-25-62$ & & & T DAVIS \\
\hline 316 & 362910 & 1162814 & 701.0 & 15.2 & & & 17.7 & 01-19-84 & & & CLEVELAND, ANETTA L. \\
\hline 317 & 362909 & 1162842 & 701.0 & 18.6 & & & 14.9 & $06-25-62$ & & & CLEVELAND, FRANK R. \\
\hline 318 & 362908 & 1163015 & 701.0 & 62.5 & & & 15.5 & $10-31-62$ & & & \\
\hline 319 & 362906 & 1161951 & 690.3 & 47.9 & 2.1 & 47.9 & 0.9 & $07-22-93$ & $\mathrm{~F}$ & SODA SPRING WELL & SPRING MEADOWS INC \\
\hline 320 & 362905 & 1160923 & 730.0 & 41.1 & 27.7 & 41.1 & 10.7 & $10-29-84$ & & & MADAME \\
\hline 321 & 362905 & 1160923 & 730.0 & 41.1 & 27.7 & 41.1 & & $01-16-87$ & $\mathbf{P}$ & & BUTLER, MIKE \\
\hline 322 & 362905 & 1162740 & 692.8 & & & & 19.3 & $01-12-87$ & & & \\
\hline 323 & 362904 & 1162808 & & & & & & $00-00-00$ & & & \\
\hline 324 & 362902 & 1163047 & 696.4 & 62.5 & & & 21.6 & $01-12-87$ & $\mathbf{R} \mathbf{R}$ & & \\
\hline 325 & 362901 & 1162028 & 690.3 & 84.7 & 3.7 & 84.7 & 0.9 & $01-21-67$ & & & NYE COUNTY LAND COMPANY \\
\hline 326 & 362858 & 1160915 & 730.7 & 25.9 & & & 11.3 & $06-19-62$ & & & \\
\hline 327 & 362857 & 1161947 & 690.9 & 101.2 & 12.2 & 101.2 & & $00-00-00$ & & ROGERS SPRING WELL 2 & SPRING MEADOWS INC. \\
\hline 328 & 362855 & 1161950 & 690.6 & 61.6 & 30.5 & 61.6 & 0.2 & $04-22-70$ & & ROGERS SPRING WELL & SPRING MEADOWS INC. \\
\hline 329 & 362850 & 1162026 & 685.8 & 195.4 & 14.6 & 213.3 & 3.7 & $03-01-67$ & & & NYE COUNTY LAND COMPANY \\
\hline 330 & 362848 & 1162646 & 690.3 & 120.7 & 18.3 & 120.7 & 18.9 & $03-16-67$ & & GILGANS NORTH WELL & STEELMAN, JAMES, C. \\
\hline
\end{tabular}




\begin{tabular}{|c|c|c|c|c|c|c|c|c|c|c|c|}
\hline $\begin{array}{l}\text { Site } \\
\text { No }\end{array}$ & Lat & Long & $\begin{array}{l}\text { Land } \\
\text { Elev }\end{array}$ & $\begin{array}{l}\text { Total } \\
\text { Depth }\end{array}$ & $\begin{array}{l}\text { Perfo } \\
\text { top }\end{array}$ & $\begin{array}{l}\text { ation } \\
\text { bottom }\end{array}$ & $\begin{array}{l}\text { Water } \\
\text { Level }\end{array}$ & $\begin{array}{l}\text { Measure } \\
\text { Date }\end{array}$ & $\begin{array}{l}\text { Note } \\
\text { O C }\end{array}$ & Site Name & Dwner \\
\hline 331 & 362840 & 1161926 & 697.9 & 146.3 & 30.5 & 144.8 & & $06-14-62$ & $\mathbf{F}$ & & NYE LAND COMPANY \\
\hline 332 & 362839 & 1162637 & & & & & & $00-00-00$ & & & \\
\hline 333 & 362836 & 1160930 & 729.5 & 42.7 & 11.9 & 42.4 & 10.1 & $10-30-61$ & & & DANIELS, JAMES M. \\
\hline 334 & 362835 & 1162641 & 690.9 & 126.5 & 16.8 & 126.5 & 17.2 & $09-29-67$ & & GILGANS SOUTH WELL & STEELMAN, JAMES C. \\
\hline 335 & 362833 & 1160206 & & & & & & $00-00-00$ & & & \\
\hline 336 & 362819 & 1161855 & 710.8 & 28.0 & & 28.0 & & $00-00-00$ & & PURGATORY SPRING WELL & SPRING MEADOWS INC. \\
\hline 337 & 362811 & 1162024 & 687.3 & 183.5 & 18.3 & 151.5 & 4.6 & $03-01-67$ & & NYE COUNTY LAND COMPANY & \\
\hline 338 & 362809 & 1162131 & 673.6 & 170.1 & 8.5 & 170.1 & 3.4 & $03-01-67$ & & & NYE COUNTY LAND COMPANY \\
\hline 339 & 362802 & 1162239 & 666.0 & 30.5 & 4.6 & 30.5 & 4.6 & $03-21-64$ & & TRENARY WELL & TRENARY, DONALD G. \\
\hline 340 & 362758 & 1162112 & 673.6 & 61.6 & 2.1 & 61.6 & 1.5 & $06-10-68$ & & & SPRING MEADOWS INC \\
\hline 341 & 362757 & 1162024 & 682.7 & 152.4 & & 91.4 & 5.2 & $05-01-71$ & & & \\
\hline 342 & 362757 & 1162024 & 682.7 & 152.4 & 30.2 & 152.4 & 6.7 & $04-21-70$ & & & SPRING MEADOWS INC \\
\hline 343 & 362755 & 1161904 & 721.5 & 37.5 & & 30.5 & & $05-02-90$ & $\mathbf{F}$ & FIVE SPRINGS WELL & SPRING MEADOWS INC. \\
\hline 344 & 362755 & 1161904 & 721.4 & 27.4 & & 27.4 & 1.8 & $06-26-68$ & & FIVE SPRINGS SHALLOW WELL & \\
\hline 345 & 362755 & 1162020 & 687.9 & 17.4 & & & 5.8 & $07-14-93$ & & MINE SHAFT & \\
\hline 346 & 362745 & 1162040 & 682.7 & 61.6 & & 61.6 & 2.4 & $06-30-68$ & & & SPRING MEADOWS INC \\
\hline 347 & 362744 & 1161243 & 709.6 & 7.6 & & & & $00-00-00$ & & AMARGOSA FLAT CORRAL WELL & \\
\hline 348 & 362743 & 1163134 & 683.9 & & & & & $00-00-00$ & & & \\
\hline 349 & 362740 & 1161210 & & & & & & $00-00-00$ & & & \\
\hline 350 & 362738 & 1161049 & 720.8 & 6.7 & & & 4.8 & $06-09-62$ & & BUCK MINING WINDMILL WELL & BUCK MINING CO. \\
\hline 351 & 362736 & 1162857 & 691.9 & 536.7 & 591.0 & 594.0 & 17.0 & $01-11-87$ & & & USGS \\
\hline 352 & 362736 & 1162857 & 691.9 & 30.5 & 29.6 & 30.5 & 15.7 & $01-11-87$ & & & USGS \\
\hline 353 & 362727 & 1163222 & 683.9 & 37.8 & & & 13.0 & $06-19-62$ & & & MORRIS \& VAN DER LENDON \\
\hline 354 & 362726 & 1163144 & 683.3 & 91.4 & & & 11.2 & $01-13-87$ & & & MORRIS \& VAN DER LENDON \\
\hline 355 & 362725 & 1163059 & 681.9 & 119.8 & & & 10.0 & $01-13-87$ & & & MORRIS \& VAN DER LENDON \\
\hline 356 & 362715 & 1162100 & 672.1 & & & & & $03-17-87$ & F & & \\
\hline 357 & 362715 & 1163223 & 685.0 & 91.4 & & & 9.8 & $08-03-86$ & & & MORRIS \& VAN DER LENDON \\
\hline 358 & 362705 & 1163223 & 684.2 & & & & 9.0 & $08-03-86$ & & & \\
\hline 359 & 362705 & 1163150 & 682.7 & & & & 9.4 & $01-11-86$ & & & \\
\hline 360 & 362705 & 1163009 & 680.0 & 7.0 & & & 6.3 & $06-18-62$ & & & \\
\hline
\end{tabular}




\begin{tabular}{|c|c|c|c|c|c|c|c|c|c|c|c|}
\hline $\begin{array}{l}\text { Site } \\
\text { No }\end{array}$ & Lat & Long & $\begin{array}{l}\text { Land } \\
\text { Elev }\end{array}$ & $\begin{array}{l}\text { Total } \\
\text { Depth }\end{array}$ & $\begin{array}{l}\text { Perfor } \\
\text { top }\end{array}$ & $\begin{array}{l}\text { ation } \\
\text { bottom }\end{array}$ & $\begin{array}{l}\text { Water } \\
\text { Level }\end{array}$ & $\begin{array}{l}\text { Measure } \\
\text { Date }\end{array}$ & $\begin{array}{l}\text { Note } \\
\text { O C }\end{array}$ & Site Name & Owner \\
\hline 361 & 362702 & 1163222 & 684.2 & 86.6 & & & 13.7 & $08-03-86$ & & & MORRIS \& VAN DER LENDON \\
\hline 362 & 362650 & 1163115 & 681.1 & 91.4 & & & 9.8 & $06-19-62$ & & & MORRIS \& VAN DER LENDON \\
\hline 363 & 362648 & 1162014 & 675.1 & 137.2 & 27.4 & 137.2 & 0.9 & $01-26-73$ & & PETERSON WELL & SPRING MEADOWS INC \\
\hline 364 & 362648 & 116274668 & 8.8 & 46.0 & 36.3 & 46.0 & & $01-15-87$ & $\mathbf{P}$ & & INDUSTRIAL MINERAL VENTURES \\
\hline 365 & 362642 & 1162125 & 661.7 & 156.7 & 45.7 & 152.4 & & $01-19-84$ & & & NYE COUNTY LAND CO. \\
\hline 366 & 362642 & 1162054 & 664.4 & & & & & $00-00-00$ & & & SPRING MEADOWS INC \\
\hline 367 & 362555 & 1162053 & 658.0 & 61.6 & 42.7 & 54.9 & 2.3 & $03-17-87$ & & GARNERS WELL & \\
\hline 368 & 362554 & 1162040 & 664.4 & 36.6 & 18.3 & 36.6 & 1.8 & $05-25-67$ & & & MERCURY FARMS INC. \\
\hline 369 & 362535 & 1162442 & & & & & & $00-00-00$ & & & \\
\hline 370 & 362532 & 1161727 & 719.3 & & & & 0.5 & $05-15-93$ & & DEVILS HOLE NR ASH MEADOWS & \\
\hline 371 & 362532 & 1161727 & 719.3 & & & & 0.6 & $05-21-93$ & & DEVILS HOLE NR ASH MEADOWS & \\
\hline 372 & 362530 & 1161715 & 732.7 & 61.0 & 14.6 & 61.0 & 14.8 & $04-15-80$ & & DEVILS HOLE WELL & NYE COUNTY LAND CO \\
\hline 373 & 362530 & 1162140 & 650.7 & & & & 0.1 & $01-15-87$ & & & \\
\hline 374 & 362529 & 1161558 & 737.6 & & & & & $12-21-93$ & O & & \\
\hline 375 & 362527 & 1161608 & 741.5 & 80.8 & & & 23.2 & $05-01-70$ & & SPRING MEADOWS 12 & SPRING MEADOWS INC. \\
\hline 376 & 362526 & 1161818 & 705.6 & 72.5 & & & 15.5 & $09-01-70$ & & SCHOOL SPRING WELL & \\
\hline 377 & 362525 & 1162743 & 664.4 & 14.3 & 6.1 & 7.0 & 3.8 & $01-13-87$ & & & USGS \\
\hline 378 & 362525 & 1162745 & 664.9 & 3.0 & & & 2.8 & $06-18-62$ & & & \\
\hline 379 & 362525 & 1162743 & 667.8 & 332.2 & 324.0 & 324.9 & 2.1 & $08-01-86$ & & NA-9 WELL & USGS \\
\hline 380 & 362521 & 1161608 & 744.3 & 65.5 & & & 29.0 & $01-01-68$ & & SPRING MEADOWS 11 & SPRING MEADOWS INC \\
\hline 381 & 362518 & 1161913 & 672.1 & 157.3 & & & & $03-24-6$ & $\mathbf{F}$ & SPRING MEADOWS 6 & \\
\hline 382 & 362517 & 1162340 & 731.5 & 6.4 & & & 4.4 & $06-16-62$ & & & \\
\hline 383 & 362459 & 1162510 & & & & & & $00-00-00$ & & & \\
\hline 384 & 362452 & 1162510 & 665.4 & 122.5 & & & 23.5 & $01-18-84$ & & & B EMBRY \\
\hline 385 & 362451 & 1162541 & & & & & & $00-00-00$ & & & \\
\hline 386 & 362443 & 1162636 & 669.0 & 0.9 & & & & $00-00-00$ & & & CALIFORNIA DIVISION OF HIGHWAYS \\
\hline 387 & 362443 & 116263 & 6658.3 & & & & 7.6 & $01-13-87$ & $\mathbf{R} \mathbf{R}$ & & \\
\hline 388 & 362434 & 1161 & 11685.2 & 85.3 & 25.0 & 85.3 & 5.5 & $09-26-69$ & & SPRING MEADOWS 9 & SPRING MEADOWS INC. \\
\hline 389 & 362432 & 1161657 & 706.7 & 152.4 & 42.4 & 152.4 & 7.3 & $02-13-70$ & & POINT OF ROCKS NORTH WELL & SPRING MEADOWS INC. \\
\hline 390 & 362425 & 1161649 & 704.1 & 61.9 & 1.2 & 61.9 & 7.6 & $07-08-68$ & & & SPRING MEADOWS INC. \\
\hline
\end{tabular}




\begin{tabular}{|c|c|c|c|c|c|c|c|c|c|c|c|}
\hline $\begin{array}{l}\text { Site } \\
\text { No }\end{array}$ & Lat & Long & $\begin{array}{l}\text { Land } \\
\text { Elev }\end{array}$ & $\begin{array}{l}\text { Total } \\
\text { Depth }\end{array}$ & $\begin{array}{l}\text { Perfo } \\
\text { top }\end{array}$ & $\begin{array}{l}\text { ation } \\
\text { bottom }\end{array}$ & $\begin{array}{l}\text { Water } \\
\text { Level }\end{array}$ & $\begin{array}{l}\text { Measure } \\
\text { Date }\end{array}$ & $\begin{array}{l}\text { Note } \\
\text { O C }\end{array}$ & Site Name & Owner \\
\hline 391 & 362420 & 1161637 & 711.2 & 178.6 & 40.2 & 142.3 & 20.9 & $04-22-70$ & & POINT OF ROCKS SOUTH WELL & NYE COUNTY LAND CO. \\
\hline 392 & 362420 & 1161637 & 711.2 & 178.6 & 40.2 & 142.3 & 20.9 & $04-22-70$ & & POINT OF ROCKS SOUTH WELL & SPRING MEADOWS INC. \\
\hline 393 & 362415 & 1162705 & 659.9 & & & & 9.2 & $01-13-87$ & & & \\
\hline 394 & 362412 & 1161545 & 713.2 & & & & 20.5 & $03-09-72$ & & & SPRING MEADOWS INC. \\
\hline 395 & 362405 & 1161539 & 708.6 & 152.4 & 30.5 & 152.4 & 1.8 & $08-27-69$ & & SPRING MEADOWS 17 & SPRING MEADOWS INC. \\
\hline 396 & 362405 & 1161545 & 709.5 & 195.7 & & 195.7 & 3.7 & $03-30-72$ & & SPRING MEADOWS 16 & SPRING MEADOWS INC. \\
\hline 397 & 362405 & 1161552 & 707.4 & 120.4 & 47.2 & 120.4 & 4.6 & $07-25-69$ & & SPRING MEADOWS 1 & SPRING MEADOWS INC. \\
\hline 398 & 362404 & 1161630 & 702.2 & 152.4 & 30.5 & 152.4 & 1.8 & $08-27-69$ & & SPRING MEADOWS 4 & SPRING MEADOWS INC. \\
\hline 399 & 362402 & 1161722 & 691.9 & 287.7 & 128.0 & 287.7 & 3.7 & $03-01-71$ & & & SPRING MEADOWS INC \\
\hline 400 & 362401 & 1161618 & 707.1 & & & & & $00-00-00$ & & & NYE COUNTY LAND COMPANY \\
\hline 401 & 362358 & 1161601 & 704.1 & & & & & $03-09-72$ & $\mathbf{P}$ & & SPRING MEADOWS INC. \\
\hline 402 & 362358 & 1161615 & 702.5 & 85.9 & 12.2 & 85.9 & & $12-01-88$ & $\mathbf{F}$ & & SPRING MEADOWS INC. \\
\hline 403 & 362357 & 1161605 & 703.4 & 126.5 & 18.3 & 91.4 & 4.9 & $05-03-69$ & & SPRING MEADOWS 2 & SPRING MEADOWS INC. \\
\hline 404 & 362356 & 1161807 & 684.2 & 259.7 & 64.0 & 256.0 & & $03-17-87$ & $\mathbf{F}$ & & NYE COUNTY LAND CO. \\
\hline 405 & 362352 & 1161552 & 703.4 & 237.7 & 3.0 & 237.7 & & $00-00-00$ & & SPRING MEADOWS 3 & SPRING MEADOWS INC. \\
\hline 406 & 362310 & 1161709 & 686.4 & 45.7 & 15.2 & 45.1 & -0.9 & $09-22-79$ & & HARRIS WELL & HARRIS, JACK \\
\hline 407 & 362303 & 1161745 & 676.6 & 4.6 & & & 1.8 & $07-23-93$ & & IMV BOREHOLE & \\
\hline 408 & 362250 & 1161750 & 673.6 & & & & 1.7 & $08-16-93$ & & HALE BOREHOLE & \\
\hline 409 & 362250 & 1162651 & 664.4 & 512.0 & 502.9 & 505.9 & 23.5 & $10-02-87$ & & & \\
\hline 410 & 362250 & 1162651 & 664.4 & 37.8 & 30.5 & 31.4 & 22.9 & $10-02-87$ & & & \\
\hline 411 & 362250 & 1161753 & 673.0 & 30.5 & 11.0 & 30.5 & 1.9 & $08-16-93$ & & HALE WELL & HALE, DEAN \\
\hline 412 & 362230 & 1163929 & 831.6 & 198.1 & 30.51 & 98.1 & 249.9 & $05-16-83$ & & TRAVERTINE POINT I WELL & US BORAX \\
\hline 413 & 362153 & 1161715 & 676.0 & 81.4 & 24.4 & 81.4 & 1.8 & $06-19-65$ & & EAST WEST MINERALS & \\
\hline 414 & 362153 & 1161715 & 676.0 & 81.4 & 24.4 & 81.4 & 1.8 & $06-19-65$ & & ASH MEADOWS LODGE & \\
\hline 415 & 362153 & 1161715 & 674.2 & 53.3 & 24.4 & 53.3 & 1.5 & $12-08-79$ & & ASH MEADOWS LODGE WELL & PENNINGTON, J. \\
\hline 416 & 362140 & 1162610 & 646.1 & 603.5 & 594.3 & 597.4 & 21.2 & $01-13-87$ & & & USGS \\
\hline 417 & 362105 & 1163128 & 703.7 & 62.8 & & 62.8 & 19.5 & $03-18-59$ & & & BARR, CHARLES M. \\
\hline 418 & 362103 & 1161600 & 699.5 & 364.8 & 355.4 & 358.4 & 4.1 & $10-29-86$ & & GS-2 DEEP & USGS \\
\hline 419 & 362103 & 1161600 & 699.5 & 36.6 & 35.7 & 36.6 & 4.0 & $10-29-86$ & & GS-2 SHALLOW & USGS \\
\hline 420 & 362054 & 1160134 & 896.7 & 131.1 & 112.8 & & 110.9 & $06-01-71$ & & & RIEHL AL W \\
\hline
\end{tabular}




\begin{tabular}{|c|c|c|c|c|c|c|c|c|c|c|c|}
\hline $\begin{array}{l}\text { Site } \\
\text { No }\end{array}$ & Lat & Long & $\begin{array}{l}\text { Land } \\
\text { Elev }\end{array}$ & $\begin{array}{l}\text { Total } \\
\text { Depth }\end{array}$ & $\begin{array}{l}\text { Perfor } \\
\text { top }\end{array}$ & $\begin{array}{l}\text { ation } \\
\text { bottom }\end{array}$ & $\begin{array}{l}\text { Water } \\
\text { Level }\end{array}$ & $\begin{array}{l}\text { Measure } \\
\text { Date }\end{array}$ & $\begin{array}{l}\text { Note } \\
\text { O C }\end{array}$ & Site Name & Owner \\
\hline 421 & 362036 & 1160150 & 868.6 & 128.3 & 88.4 & 128.3 & $92.0 \quad C$ & $02-17-76$ & & & NOBLE KAY \\
\hline 422 & 362033 & 1162552 & 639.4 & & & & $11.3 \mathrm{C}$ & $01-13-87$ & & & \\
\hline 423 & 362033 & 1162552 & 640.0 & 5.8 & & & & $00-00-00$ & & & \\
\hline 424 & 362021 & 1161330 & 740.7 & 481.6 & 472.1 & 475.2 & 26.7 & $10-29-86$ & & GS-1 WELL & USGS \\
\hline 425 & 362021 & 1161330 & 740.7 & 36.6 & & & 24.8 & $01-14-87$ & & & USGS \\
\hline 426 & 361957 & 1161752 & 716.6 & 609.6 & 600.1 & 603.2 & 64.7 & $10-29-86$ & & GS-3 WELL & USGS \\
\hline 427 & 361957 & 1161752 & 716.6 & 48.8 & 47.9 & 48.8 & 25.1 & $08-23-93$ & & GS-3 SHALLOW & USGS \\
\hline 428 & 361909 & 1160134 & 811.6 & 227.4 & & & 33.6 & $02-25-75$ & & & CNUC WELL 41 \\
\hline 429 & 361908 & 1160134 & 811.3 & & & & 36.4 & $02-17-76$ & s & & PREF.EQUITIES \\
\hline 430 & 361907 & 1160135 & 810.1 & & & & 33.6 & $03-08-77$ & & & \\
\hline 431 & 361903 & 1160120 & 812.3 & 234.7 & & & 35.3 & $02-17-76$ & & & TRI STATE \\
\hline 432 & 361900 & 1160308 & 810.4 & 45.7 & & & 34.1 & $02-17-76$ & & & GREGORY LESLIE H \\
\hline 433 & 361857 & 1160231 & 808.0 & 143.2 & 36.6 & 96.0 & 31.8 & $02-17-76$ & & & CNUC WELL 43 \\
\hline $\begin{array}{l}434 \\
435\end{array}$ & 361853 & 1160156 & 808.6 & 114.3 & 61.0 & 114.3 & 31.6 & $02-25-75$ & & & CNUC WELL 42 \\
\hline 435 & 361850 & 1161938 & 679.7 & 35.1 & & & 29.8 & $12-20-93$ & & GA-8C & USGS \\
\hline 436 & 361850 & 1161938 & 679.7 & 66.1 & & & 30.0 & $12-20-93$ & & GA-8D & USGS \\
\hline 437 & 361850 & 1161935 & 679.7 & 58.2 & & & 31.5 & $12-20-93$ & & GA-8E & USGS \\
\hline 438 & 361850 & 1161933 & 679.7 & 76.8 & & & 33.6 & $12-20-93$ & & GA-8F & USGS \\
\hline 439 & 361850 & 1161933 & 679.7 & 33.5 & & & 33.3 & $12-20-93$ & & GA-8M & USGS \\
\hline 440 & 361849 & 1161942 & 679.7 & 64.9 & & & 27.7 & $12-20-93$ & & GA-8B & USGS \\
\hline 441 & 361845 & 1161939 & 676.6 & 32.6 & & & 29.7 & $12-20-93$ & & GA-8K & USGS \\
\hline 442 & 361845 & 1161937 & 676.6 & 25.9 & & & 25.8 & $12-20-93$ & & GA-8J RATHOLE & \\
\hline 443 & 361845 & 1161937 & 676.6 & 30.3 & & & & $12-20-93$ & D I & GA-8J & \\
\hline 444 & 361835 & 1162245 & 620.8 & 2.1 & & & 1.4 & $06-19-62$ & & & CALIFORNIA DIVISION OF HIGHWAYS \\
\hline 445 & 361833 & 1162200 & 621.8 & & & & & $00-00-00$ & & WELL-15 & \\
\hline 446 & 361833 & 1162202 & 619.6 & 8.5 & & & & $12-22-93$ & F & FRANKLIN LAKE 1 & CALIFORNIA DIVISION OF HIGHWAYS \\
\hline 447 & 361826 & 1160228 & 802.8 & 139.3 & & & 26.7 & $02-17-76$ & & & CNUC WELL 44 \\
\hline 448 & 361820 & 1162513 & 624.5 & 48.8 & & & 2.7 & 03-18-87 & & & CALIFORNIA DIVISION OF HIGHWAYS \\
\hline 449 & 361817 & 1162447 & 622.3 & 68.6 & 48.8 & 61.0 & 1.0 & $01-22-79$ & & DEATH VALLEY JCT WELL & ETTIE, LEE \\
\hline 450 & 361808 & 1162735 & 668.1 & 41.1 & & & 39.5 & $03-18-87$ & & & AMERICAN BORATE CORP. \\
\hline
\end{tabular}




\begin{tabular}{|c|c|c|c|c|c|c|c|c|c|c|}
\hline $\begin{array}{l}\text { Site } \\
\text { No }\end{array}$ & Lat & Long & $\begin{array}{l}\text { Land } \\
\text { Elev }\end{array}$ & $\begin{array}{l}\text { Total } \\
\text { Depth }\end{array}$ & $\begin{array}{l}\text { Perfo } \\
\text { top }\end{array}$ & $\begin{array}{l}\text { ation } \\
\text { bottom }\end{array}$ & $\begin{array}{l}\text { Water } \\
\text { Level }\end{array}$ & $\begin{array}{l}\text { Measure } \\
\text { Date }\end{array}$ & $\begin{array}{l}\text { Note } \\
\text { O C }\end{array}$ & Site Name \\
\hline 451 & 361802 & 1160016 & 811.9 & 120.4 & & & 32.4 & $02-17-76$ & & \\
\hline 452 & 361801 & 1160017 & 811.0 & 46.0 & & & 31.5 & $02-17-76$ & & \\
\hline 453 & 361800 & 1162443 & 621.2 & 21.0 & & & $1.1 \mathrm{C}$ & $01-14-87$ & $\mathbf{R}$ & \\
\hline 454 & 361800 & 1162450 & 620.8 & 42.4 & & & $0.7 \mathrm{C}$ & $06-19-62$ & & \\
\hline 455 & 361753 & 1160009 & 813.2 & 120.4 & & & 31.9 & $12-16-93$ & & STARVER \\
\hline 456 & 361745 & 1162155 & 618.1 & 2.1 & & & $0.5 \mathrm{C}$ & $06-21-62$ & & \\
\hline 457 & 361745 & 1162155 & 618.1 & & & & & $00-00-00$ & & \\
\hline 458 & 361741 & 1160010 & 811.3 & 314.5 & & & 31.5 & $02-17-76$ & & \\
\hline 459 & 361734 & 1160122 & 799.5 & 214.0 & & & 21.5 & $02-17-76$ & & \\
\hline 460 & 361729 & 1160055 & 804.0 & 246.3 & 70.7 & 155.4 & 24.9 & $03-17-76$ & & \\
\hline 461 & 361724 & 1163242 & 823.9 & 609.6 & 600.1 & 603.2 & 116.3 & $08-04-86$ & & S-1 WEL \\
\hline 462 & 361724 & 1163242 & 819.9 & 137.2 & 134.1 & 137.2 & 121.2 & $01-13-8$ & & \\
\hline 463 & 361723 & 1160003 & 811.3 & 164.6 & & & 30.9 & $02-17-76$ & & \\
\hline 464 & 361718 & 1162238 & 616.3 & 17.1 & & & & $01-14-87$ & $\mathbf{F}$ & WELL-16 \\
\hline 465 & 361709 & 1160124 & 795.8 & 106.7 & & & 20.6 & $02-16-76$ & & \\
\hline 466 & 361709 & 1160051 & 800.4 & 182.9 & 30.5 & & 13.4 & $06-18-55$ & & \\
\hline 467 & 361709 & 1160042 & 802.2 & 144.8 & 21.3 & 144.8 & 20.4 & $02-04-62$ & & \\
\hline 468 & 361705 & 1162135 & 614.1 & 2.4 & & & -1.2 & $08-24-62$ & & \\
\hline 469 & 361704 & 1160303 & 787.3 & & & & 12.0 & $02-18-76$ & & \\
\hline 470 & 361703 & 1162150 & 613.7 & 0.9 & & & . & $06-21-62$ & $\mathbf{F} \mathbf{F}$ & \\
\hline 471 & 361700 & 1162202 & 615.6 & 2.4 & & & 1.0 & $07-09-86$ & & GS-9 \\
\hline 472 & 361700 & 1162202 & 616.9 & 4.9 & & & -0.6 & $07-09-86$ & & GS-10 \\
\hline 473 & 361700 & 1162202 & 614.4 & 1.8 & & & 0.8 & $07-09-86$ & & GS-11 \\
\hline 474 & 361700 & 1162202 & 615.4 & 8.8 & & & -2.3 & $06-24-83$ & $\mathbf{G}$ & GS-8 \\
\hline 475 & 361647 & 1160439 & 785.1 & 19.5 & & & 10.5 & $03-08-77$ & & \\
\hline 476 & 361642 & 1160123 & 795.5 & 152.4 & & & 14.6 & $04-03-61$ & & \\
\hline 477 & 361640 & 1163155 & 613.1 & 2.7 & & & -0.2 & $08-24-62$ & & \\
\hline 478 & 361638 & 1160437 & 785.4 & 10.5 & & & 6.5 & $02-04-62$ & & \\
\hline 479 & 361636 & 1160009 & 805.9 & 335.9 & & & 6.9 & $11-26-52$ & & \\
\hline 480 & 361627 & 1162212 & 612.9 & 8.3 & & & -1.6 & $07-09-86$ & & GS-13 \\
\hline
\end{tabular}

Owner

STRAVER-DAWSON
STRAVER-DAWSON
LEE, E.
LEE, E.
STARVER, DAWSON

CALIFORNIA DIVISION OF HIGHWAY CALIFORNIA DIVISION OF HIGHWAYS STRAVER-DAWSON WOLFSON-FOWLER WOLFSEN

STAVERS

BELLS ALVIN

BELLS ALVIN

FOWLER H M

CALIFORNIA DIVISION OF HIGHWAYS

CALIFORNIA DIVISION OF HIGHWAYS

USGS

BELLS ALVIN

CALIFORNIA DIVISION OF HIGHWAYS USGS

BELLS ALVIN 


\begin{tabular}{|c|c|c|c|c|c|c|c|c|c|c|c|}
\hline $\begin{array}{l}\text { Site } \\
\text { No }\end{array}$ & Lat & Long & $\begin{array}{l}\text { Land } \\
\text { Elev }\end{array}$ & $\begin{array}{l}\text { Total } \\
\text { Depth }\end{array}$ & $\begin{array}{l}\text { Perfor } \\
\text { top }\end{array}$ & $\begin{array}{l}\text { ation } \\
\text { bottom }\end{array}$ & $\begin{array}{l}\text { Water } \\
\text { Level }\end{array}$ & $\begin{array}{l}\text { Measure } \\
\text { Date }\end{array}$ & $\begin{array}{l}\text { Note } \\
\text { O C }\end{array}$ & Site Name & Owner \\
\hline 481 & 361627 & 1162212 & 612.9 & 6.4 & & & -0.1 & $07-09-86$ & & GS-14 & \\
\hline 482 & 361627 & 1162212 & 612.9 & 8.8 & & & 0.9 & $07-09-86$ & & GS-12 & \\
\hline 483 & 361616 & 1160125 & 794.6 & 61.0 & & & 12.9 & $02-04-62$ & & & CADWELL H. \\
\hline 484 & 361616 & 1160003 & 807.7 & 199.6 & & & 24.5 & $02-17-76$ & & & BELLS ALVIN \\
\hline 485 & 361615 & 116002 & 6801.6 & 163.7 & & & 21.0 & $02-17-76$ & & & BELLS ALVIN \\
\hline 486 & 361612 & 1160051 & 807.7 & 155.4 & & & 19.4 & $02-17-76$ & & & BELLS ALVIN \\
\hline 487 & 361610 & 1162237 & 611.6 & 7.3 & & & 4.0 & $03-22-86$ & & & GS-19 \\
\hline 488 & 361608 & 1160441 & 783.3 & 155.4 & 18.3 & 155.4 & 9.7 & $02-17-76$ & & & BELLS ALVIN \\
\hline 489 & 361606 & 1162127 & 611.7 & 3.7 & & & 0.5 & $03-21-86$ & & GS-2 & \\
\hline 490 & 361605 & 1162127 & 612.0 & 2.1 & & & 0.5 & $03-21-86$ & & GS-1 & \\
\hline 491 & 361553 & 1162121 & 611.4 & 6.8 & & & 1.2 & $07-09-86$ & & GS-4 & \\
\hline 492 & 361553 & 1162121 & 611.5 & 6.5 & & & 0.2 & $07-09-86$ & & GS-5 & \\
\hline 493 & 361553 & 1162121 & 611.4 & 1.6 & & & 1.2 & $07-09-86$ & & GS-6 & \\
\hline 494 & 361553 & 1162121 & 612.5 & 8.2 & & & -0.5 & $07-09-86$ & & GS-7 & \\
\hline 495 & 361553 & 1162121 & 611.4 & 9.8 & & & -0.3 & $07-09-86$ & & GS-3 & \\
\hline 496 & 361552 & 1160009 & 803.4 & 261.2 & 73.1 & & 18.9 & $02-17-76$ & & & BELLS ALVIN \\
\hline 497 & 361548 & 1160054 & 796.7 & 157.0 & & & 16.5 & $02-18-76$ & & & MCCOWAN J. \\
\hline 498 & 361548 & 1160145 & 787.6 & 153.6 & & & 10.7 & $02-18-76$ & & & LEWIS WARREN \\
\hline 499 & 361536 & 1160051 & 794.9 & 235.6 & & & 14.9 & $03-18-76$ & & & BLOSSER E. \\
\hline 500 & 361533 & 1161119 & 748.9 & 8.2 & & & 6.2 & $01-28-76$ & & & USGS \\
\hline 501 & 361530 & 1160010 & 137.2 & & & & & $00-00-00$ & & WILCOX WELL & \\
\hline 502 & 361530 & 1160301 & 782.4 & & & & 8.3 & $02-18-76$ & & & \\
\hline 503 & 361525 & 1160018 & 799.1 & 217.0 & & & & $03-15-53$ & F & & BLOSSER T. \\
\hline 504 & 361523 & 1160051 & 794.6 & 236.2 & & & & $03-18-87$ & $\mathbf{P}$ & HARMER & HARMER, R. S. \\
\hline 505 & 361523 & 1160156 & 787.6 & 262.1 & & & 6.2 & $02-04-62$ & & & SANDSTEAD F W \\
\hline 506 & 361522 & 1160447 & 782.1 & 152.4 & 91.4 & 152.4 & 8.7 & $02-18-76$ & & & BELLS ALVIN \\
\hline 507 & 361522 & 1160123 & 790.3 & 85.3 & 73.1 & & 10.7 & $02-20-73$ & & & BLOSSER T. \\
\hline 508 & 361517 & 1163224 & 853.4 & & & & 20.0 & $01-09-86$ & & & AMERICAN BORATE CORP. \\
\hline 509 & 361517 & 1162201 & 610.5 & 1.2 & & & & $00-00-00$ & & & CALIFORNIA DIVISION OF HIGHWAYS \\
\hline 510 & 361516 & 1162201 & 610.7 & 3.3 & & & 2.0 & $07-10-86$ & & GS-16 & \\
\hline
\end{tabular}




\begin{tabular}{|c|c|c|c|c|c|c|c|c|c|c|c|}
\hline $\begin{array}{l}\text { Site } \\
\text { No }\end{array}$ & Lat & Long & $\begin{array}{l}\text { Land } \\
\text { Elev }\end{array}$ & $\begin{array}{l}\text { Total } \\
\text { Depth }\end{array}$ & $\begin{array}{l}\text { Perfor } \\
\text { top }\end{array}$ & $\begin{array}{l}\text { ation } \\
\text { bottom }\end{array}$ & $\begin{array}{l}\text { Water } \\
\text { Level }\end{array}$ & $\begin{array}{l}\text { Measure } \\
\text { Date }\end{array}$ & $\begin{array}{l}\text { Note } \\
\text { O C }\end{array}$ & Site Name & Owner \\
\hline 511 & 361516 & 1162201 & 610.6 & 9.8 & & & 1.9 & $07-10-86$ & & GS-17 & \\
\hline 512 & 361516 & 1162201 & 610.6 & 6.9 & & & 1.9 & $07-10-86$ & & GS-15 & \\
\hline 513 & 361513 & 1160550 & 789.4 & 30.5 & & & 14.8 & $02-17-76$ & & & LASS CARL \\
\hline 514 & 361513 & 1163131 & 829.0 & & & & 31.3 & $01-09-86$ & & & AMERICAN BORATE CORP. \\
\hline 515 & 361512 & 1160532 & 785.1 & 13.4 & & & 11.6 & $02-17-76$ & & & USGS \\
\hline 516 & 361512 & 1160532 & 784.8 & 9.8 & & & 7.8 & $02-04-62$ & & & USGS \\
\hline 517 & 361455 & 1160349 & 779.6 & 36.6 & & & 7.0 & $02-18-76$ & & & JOHNSON H A \\
\hline 518 & 361451 & 1160025 & 801.6 & 251.4 & 51.2 & & 13.1 & $02-18-76$ & & & SHURTLIFF L N \\
\hline 519 & 361449 & 1160442 & 779.9 & & & & 7.6 & $02-18-76$ & & & \\
\hline 520 & 361446 & 1160103 & 794.0 & & & & 14.4 & $02-18-76$ & & & \\
\hline 521 & 361446 & 1160056 & 795.2 & 206.6 & 79.2 & & 9.7 & $02-18-76$ & & & MURPHY D J \\
\hline 522 & 361444 & 1162157 & 610.3 & 8.2 & 7.9 & 8.2 & 2.8 & $07-10-86$ & & GS-18 & \\
\hline 523 & 361443 & 1162331 & 610.9 & 4.6 & & & 3.1 & $07-10-86$ & & WELL-13 & \\
\hline 524 & 361429 & 1160018 & 797.3 & 234.4 & 58.5 & & 11.1 & $02-18-76$ & & & PECHSTEIN \\
\hline 525 & 361429 & 1160049 & 793.7 & 226.2 & & & 13.1 & $02-18-76$ & & & PECHSTEIN E B \\
\hline 526 & 361417 & 1162240 & 609.6 & 12.2 & & & 3.2 & $03-13-86$ & & & \\
\hline 527 & 361415 & 1160918 & 750.7 & 61.0 & & & 5.5 & $01-18-76$ & & & COHEN ROBERT \\
\hline 528 & 361415 & 1162221 & 609.5 & 10.7 & & & 1.9 & $07-10-86$ & & WELL- 5 & \\
\hline 529 & 361415 & 1162221 & 609.6 & 8.2 & & & 2.3 & $06-14-85$ & & & \\
\hline 530 & 361415 & 1162221 & 609.6 & 4.6 & & & 1.4 & $06-14-85$ & & & \\
\hline 531 & 361415 & 1162221 & 609.5 & 10.7 & & & 1.9 & $07-10-86$ & & & \\
\hline 532 & 361415 & 1160926 & 755.0 & & & & 6.7 & $08-23-93$ & & & \\
\hline 533 & 361415 & 1162302 & 610.2 & 18.0 & & & 1.4 & $07-10-86$ & & WELL-14 & \\
\hline 534 & 361413 & 1162212 & 609.1 & 6.1 & & & 3.2 & 06-14-85 & & & \\
\hline 535 & 361413 & 1162212 & 609.6 & 6.1 & & & 3.2 & $03-18-86$ & & & \\
\hline 536 & 361413 & 1162212 & 609.6 & 9.4 & & & 2.9 & $06-14-85$ & & & \\
\hline 537 & 361412 & 1162230 & 607.7 & 12.2 & & & 2.3 & $07-10-86$ & & WELL-10 & \\
\hline 538 & 361411 & 1161003 & 748.9 & 8.2 & & & 5.3 & $01-28-76$ & & & USGS \\
\hline 539 & 361409 & 1160639 & 795.5 & 201.8 & & & & $02-04-62$ & $\mathbf{F}$ & & LOTTERER BLAGG \\
\hline 540 & 361408 & 1160918 & 749.8 & 51.8 & & & 2.6 & $01-28-76$ & & & \\
\hline
\end{tabular}




\begin{tabular}{|c|c|c|c|c|c|c|c|c|c|c|c|}
\hline $\begin{array}{l}\text { Site } \\
\text { No }\end{array}$ & Lat & Long & $\begin{array}{l}\text { Land } \\
\text { Elev }\end{array}$ & $\begin{array}{l}\text { Total } \\
\text { Depth }\end{array}$ & $\begin{array}{l}\text { Perfor } \\
\text { top }\end{array}$ & $\begin{array}{l}\text { ation } \\
\text { bottom }\end{array}$ & $\begin{array}{l}\text { Water } \\
\text { Level }\end{array}$ & $\begin{array}{l}\text { Measure } \\
\text { Date }\end{array}$ & $\begin{array}{l}\text { Note } \\
\text { O C }\end{array}$ & Site Name & Owner \\
\hline 541 & 361406 & 1161106 & 749.5 & 12.8 & & & 7.1 & $01-28-76$ & & & USGS \\
\hline 542 & 361405 & 1160332 & 779.6 & 61.0 & 9.1 & 51.2 & 11.0 & $12-16-93$ & & ROOKRIDGE AND CARRADO & ROOKRIDGE \& CARRADO \\
\hline 543 & 361401 & 1160421 & 777.5 & 61.0 & & & 5.0 & $02-03-62$ & & & STUB I A \\
\hline 544 & 361355 & 1160904 & 751.3 & 36.6 & & & 3.7 & $01-28-76$ & & & LYON CHALMER \\
\hline 545 & 361352 & 1160053 & 791.8 & & & & 12.5 & $02-18-76$ & & & \\
\hline 546 & 361334 & 1160156 & 786.3 & 118.9 & & & 9.1 & $02-18-76$ & & & CADWELL H L \\
\hline 547 & 361333 & 1160509 & 775.4 & 60.3 & 9.1 & & 6.5 & $02-03-62$ & & & BONANITO J \\
\hline 548 & 361331 & 1160036 & 793.4 & & & & 11.5 & $02-18-76$ & & & \\
\hline 549 & 361331 & 1160018 & 795.8 & 304.8 & 33.5 & & 7.7 & $02-04-62$ & & & LOTTERER JACK J \\
\hline 550 & 361327 & 1162249 & 608.5 & 4.5 & & & 3.9 & $07-10-86$ & & GS-20 & \\
\hline 551 & 361327 & 1160018 & 795.5 & 198.1 & 61.0 & & -8.0 & $02-04-62$ & & & LOTTERER J F \\
\hline 552 & 361323 & 1160304 & 779.9 & 48.8 & 24.4 & 48.8 & 9.1 & $02-09-82$ & & ANCHOR INN MOBILE HOME PRK & K ANDERSON, BERNARD \\
\hline 553 & 361321 & 1160418 & 776.6 & & & & 7.5 & $02-18-76$ & & & \\
\hline 554 & 361318 & 1160155 & 784.8 & 30.5 & 15.2 & 30.5 & 6.7 & $12-08-68$ & & RANCHO VISTA NO 3 & WULFENSTEIN, RAY \\
\hline 555 & 361315 & 1160213 & 785.1 & & & & & $00-00-00$ & & SUNSET MOBILE HOME PARK & \\
\hline 556 & 361315 & 1160208 & 783.0 & & & & & $00-00-00$ & & SUNSET MOBILE HOME PARK & \\
\hline 557 & 361313 & 1160612 & 777.2 & 22.9 & & & 13.2 & $02-20-76$ & & & USGS \\
\hline 558 & 361311 & 1160255 & 780.9 & & & & 8.9 & $02-18-76$ & & & \\
\hline 559 & 361308 & 1160105 & 789.4 & 121.9 & & & 5.5 & $02-18-76$ & & & OWENS BOB \\
\hline 560 & 361308 & 1160124 & 788.8 & 121.9 & & & 7.8 & $02-27-75$ & & & KELLY JOE \\
\hline 561 & 361308 & 1160052 & 791.2 & 128.6 & & & 7.8 & $02-18-76$ & & & ROOT EW \\
\hline 562 & 361306 & 1160332 & 776.9 & 45.7 & 18.3 & 45.7 & 8.5 & $04-10-75$ & & BASIN PARK RANCHOS & WULFENSTEIN, RAY \\
\hline 563 & 361249 & 1162243 & & & & & & $00-00-00$ & & SP-50 & \\
\hline 564 & 361248 & 1161138 & 748.9 & 10.4 & 10.1 & & 7.1 & $01-28-76$ & & & USGS \\
\hline 565 & 361246 & 1160300 & 781.5 & 243.8 & & & 11.6 & $06-05-74$ & & & HORGAN \& \\
\hline 566 & 361246 & 1160331 & 777.8 & 76.2 & & & 8.8 & $02-18-76$ & & & LACOMP \\
\hline 567 & 361246 & 1160300 & 781.5 & 243.8 & & & 6.2 & $02-17-54$ & & & LYNSH CHARLES \\
\hline 568 & 361242 & 1160042 & 790.9 & & & & 8.5 & $02-28-75$ & & & RUID BOB \\
\hline 569 & 361241 & 1160114 & 787.9 & & & & & $00-00-00$ & & BIG VALLEY MOBILE HOME PRK & BIG VALLEY MOBILE HOMF \\
\hline 570 & 361231 & 1160229 & 782.7 & 41.1 & & & 7.1 & $02-04-62$ & & & FORD S \\
\hline
\end{tabular}




\begin{tabular}{|c|c|c|c|c|c|c|c|c|c|c|}
\hline $\begin{array}{l}\text { Site } \\
\text { No }\end{array}$ & Lat & Long & $\begin{array}{l}\text { Land } \\
\text { Elev }\end{array}$ & $\begin{array}{l}\text { Total } \\
\text { Depth }\end{array}$ & $\begin{array}{l}\text { Perfor } \\
\text { top }\end{array}$ & $\begin{array}{l}\text { ation } \\
\text { bottom }\end{array}$ & $\begin{array}{l}\text { Water } \\
\text { Level }\end{array}$ & $\begin{array}{l}\text { Measure } \\
\text { Date }\end{array}$ & $\begin{array}{l}\text { Note } \\
\text { O C }\end{array}$ & Site Name \\
\hline 571 & 361225 & 1160108 & 787.9 & & & & & $00-00-00$ & & BIG FIVE TRAILER PARK \\
\hline 572 & 361222 & 1160300 & 779.6 & 67.1 & & & 8.7 & $02-18-76$ & & \\
\hline 573 & 361222 & 1160044 & 790.9 & & & & 8.0 & $02-18-76$ & & \\
\hline 574 & 361218 & 1160919 & 749.8 & 9.1 & & & 3.3 & $03-30-60$ & & \\
\hline 575 & 361218 & 1160051 & 790.3 & 160.0 & 30.5 & & 5.0 & $02-18-76$ & & \\
\hline 576 & 361217 & 1160210 & 781.8 & 274.9 & & & 6.7 & $02-04-62$ & & \\
\hline 577 & 361217 & 1160100 & 797.9 & 106.7 & 12.2 & & 7.5 & $02-18-76$ & & \\
\hline 578 & 361216 & 1160537 & 774.2 & 167.9 & 18.3 & & 11.6 & $02-18-76$ & & \\
\hline 579 & 361216 & 1160609 & 771.4 & 152.4 & 11.0 & & 16.1 & $02-18-76$ & & \\
\hline 580 & 361213 & 1160155 & 785.7 & 21.3 & & & 7.1 & $02-04-62$ & & \\
\hline 581 & 361213 & 1160155 & 785.7 & 5.5 & & & 5.0 & $03-23-54$ & & \\
\hline 582 & 361210 & 1162257 & 630.9 & 39.6 & 36.6 & 39.6 & 23.4 & $01-14-87$ & & \\
\hline 583 & 361210 & 1162257 & 630.9 & & 417.6 & 423.7 & 24.0 & $01-14-87$ & & \\
\hline 584 & 361209 & 1160614 & 780.2 & 91.4 & 11.0 & 91.4 & 19.8 & $12-16-93$ & & TUDOR \\
\hline 585 & 361204 & 1160603 & 771.4 & 152.4 & 9.8 & 152.4 & 17.1 & $12-16-93$ & & TURNER \\
\hline 586 & 361158 & 1162405 & 673.6 & 106.7 & 103.6 & 106.7 & 94.0 & $01-14-87$ & & \\
\hline 587 & 361158 & 1162405 & 673.6 & 426.7 & 417.6 & 423.7 & 89.5 & $01-14-87$ & & \\
\hline 588 & 361150 & 1160350 & 775.1 & & & & & $00-00-00$ & & RANCHO VISTA NO 4-1 \\
\hline 589 & 361149 & 1160021 & 798.5 & 106.7 & & & 3.0 & $02-19-76$ & & \\
\hline 590 & 361146 & 1160428 & 774.8 & & & & 8.4 & $02-18-76$ & & \\
\hline 591 & 361145 & 1160346 & 775.1 & 61.0 & 36.6 & 54.9 & 14.9 & $01-23-93$ & & RANCHO VISTA NO 4-2 \\
\hline 592 & 361118 & 1160043 & 792.4 & 106.7 & 21.9 & & 1.7 & $02-19-76$ & & \\
\hline 593 & 361034 & 1160459 & 768.1 & 61.0 & 7.9 & & 9.5 & $02-18-76$ & & \\
\hline 594 & 361031 & 1160516 & 767.4 & 16.5 & & & 7.0 & $02-07-62$ & & \\
\hline 595 & 361030 & 1160517 & 767.4 & & & & 9.1 & $02-20-76$ & & \\
\hline 596 & 361030 & 1160143 & 783.9 & 9.1 & & & 6.1 & $02-19-76$ & & \\
\hline 597 & 361029 & 1160141 & 784.2 & 60.3 & & & 6.4 & $02-03-62$ & & \\
\hline 598 & 361014 & 1160442 & 768.7 & 77.1 & & & 10.7 & $02-18-76$ & & \\
\hline 599 & 361012 & 1160447 & 768.1 & 77.1 & 7.6 & 38.1 & 7.3 & $09-28-51$ & & WHITE \\
\hline 600 & 361012 & 1160447 & 768.1 & 77.1 & 7.6 & 38.1 & 14.2 & $12-16-93$ & & WHITE \\
\hline
\end{tabular}

Owner

\section{FORD STANLEY}

USGS

WILLIAMS W J

HORGAN L A WILLIAMS W. T WOODS R L TUDOR H D HORGAN

HORGAN L A

TUDOR, H. D. TURNER, W. M.

WULFENSTEIN, RAY WILLIAMS $W$. $T$

WULFENSTEIN, RAY WILLIAMS $W$. $T$ NV HWY DPT CRUCIE J J COLLINS FRED

GORDON C. $\mathbf{R}$ FORD J W WHITE, JOHN A. FORD, J. 


\begin{tabular}{|c|c|c|c|c|c|c|c|c|c|c|}
\hline $\begin{array}{l}\text { Site } \\
\text { No }\end{array}$ & Lat & Long & $\begin{array}{l}\text { Land } \\
\text { Elev }\end{array}$ & $\begin{array}{l}\text { Total } \\
\text { Depth }\end{array}$ & $\begin{array}{l}\text { Perforation } \\
\text { top bottom }\end{array}$ & $\begin{array}{l}\text { Water } \\
\text { Level }\end{array}$ & $\begin{array}{l}\text { Measure } \\
\text { Date }\end{array}$ & $\begin{array}{l}\text { Note } \\
\text { O C }\end{array}$ & Site Name & Owner \\
\hline 601 & 360951 & 1160722 & 754.6 & 14.3 & 13.7 & 10.7 & $04-17-63$ & & & USGS \\
\hline 602 & 360930 & 1160520 & 761.0 & 12.8 & & 7.1 & $03-07-77$ & & & USGS \\
\hline 603 & 360930 & 1160520 & 761.0 & 6.7 & & 5.7 & $02-03-62$ & & & USBLM \\
\hline 604 & 360845 & 1160225 & 773.8 & 17.4 & & 13.1 & $02-03-62$ & & & USGS \\
\hline 605 & 360845 & 1160225 & 773.8 & 19.5 & & 15.8 & $03-07-77$ & & & USGS \\
\hline 606 & 360756 & 1160035 & 787.3 & 19.5 & & 13.2 & $03-07-77$ & & & USGS \\
\hline 607 & 360751 & 1160559 & 758.3 & 31.1 & 30.5 & 18.1 & $02-05-62$ & & & USGS \\
\hline 608 & 360748 & 1160523 & 758.6 & & & 18.4 & $03-07-77$ & & & BOWMAN OSCAR \\
\hline 609 & 360702 & 1160335 & 764.7 & 25.9 & 25.3 & 16.3 & $02-20-76$ & & & USGS \\
\hline 610 & 360445 & 1160154 & 765.0 & 23.5 & 22.9 & 17.3 & $01-23-76$ & & & USGS \\
\hline 611 & 360232 & 1160036 & 765.3 & 61.0 & 60.7 & 35.6 & $11-01-77$ & & & USGS \\
\hline 612 & 363532 & 1162254 & 772.9 & 446.8 & & & & & FELDERHOFF-FEDERAL 5-1 & US BLM \\
\hline 613 & 363707 & 1162426 & 783.9 & 1530.0 & & & & & FELDERHOFF-FEDERAL 25-1 & US BLM \\
\hline
\end{tabular}




\section{APPENDIX B}

\section{POTENTIAL HYDROLOGIC CHARACTERIZATION RECOMPLETION WELLS}

Appendix water level codes under the heading descriptor:

$$
\begin{aligned}
& \text { O - original } \\
& \text { C - current }
\end{aligned}
$$

Codes:

$$
\begin{aligned}
& \text { D - dry } \\
& \text { E - recently flowing } \\
& \text { F - flowing } \\
& \text { G - nearby site flowing } \\
& \text { H - nearby site recently flowing } \\
& \text { I - injection of water } \\
& \text { J - nearby site injected with water } \\
& \text { N - measurement discontinued } \\
& \text { O - obstruction above water surface } \\
& \text { P - pumping } \\
& \text { R - recently pumped } \\
& \text { S - nearby site pumping } \\
& \text { T - nearby site recently pumping } \\
& \text { V - foreign substance on water surface } \\
& \text { W - well destroyed } \\
& \text { X - surface-water affects } \\
& \text { Z - other (explain in remarks) }
\end{aligned}
$$

Note: Latitude and Longitude (deg, $\mathrm{min}, \mathrm{sec}$ )

Elevation ( $m$ above mean sea level)

Total Depth, Perforation Interval and Water Level ( $\mathrm{m}$ below land surface)

Date (month/day/year) 


\begin{tabular}{|c|c|c|c|c|c|c|c|c|c|c|c|}
\hline $\begin{array}{l}\text { Site } \\
\text { No }\end{array}$ & Lat & Long & $\begin{array}{l}\text { Land } \\
\text { Elev }\end{array}$ & $\begin{array}{l}\text { Total } \\
\text { Depth }\end{array}$ & $\begin{array}{l}\text { Perfo } \\
\text { top }\end{array}$ & $\begin{array}{l}\text { ation } \\
\text { bottom }\end{array}$ & $\begin{array}{l}\text { Water } \\
\text { Level }\end{array}$ & $\begin{array}{l}\text { Measure } \\
\text { Date }\end{array}$ & $\begin{array}{l}\text { Note } \\
\text { O C }\end{array}$ & Site Name & Owner \\
\hline 6 & 365520 & 1163703 & 1198.1 & 487.7 & 243.8 & 487.7 & 201.2 & 09-01-89 & & GEXA WELL 4 & USNGS GEXA \\
\hline 8 & 365445 & 1163839 & 1243.8 & 213.3 & 63.4 & 213.3 & 41.1 & $09-18-88$ & & GEXA WELL 3 & GOLD GEXA \\
\hline 14 & 365128 & 1164920 & & & & & & $00-00-00$ & & ETH I-4 & \\
\hline 15 & 365106 & 1164932 & & 364.2 & & & 219.1 & $08-10-88$ & & II-1 & \\
\hline 16 & 365105 & 1164819 & & & & & & $00-00-00$ & & III-2 & \\
\hline 19 & 364814 & 1164854 & 957.0 & 457.2 & 152.4 & 457.2 & 165.9 & $01-11-87$ & & & USGS \\
\hline 20 & 364758 & 1163317 & 976.8 & & & & 187.4 & $03-10-85$ & & USW VH-1 & USGS-WRD \\
\hline 21 & 364732 & 1163307 & 963.5 & 762.3 & 277.7 & 762.3 & 184.1 & $12-23-93$ & $\mathbf{Z}$ & USW VH-1 & U.S. GOVT \\
\hline 30 & 364600 & 1165112 & 1011.9 & 127.4 & & & & $01-11-87$ & D & & US BORAX \\
\hline 31 & 364559 & 1165219 & 1146.0 & 144.8 & & & 144.6 & $10-30-86$ & & & US BORAX \\
\hline 32 & 364557 & 1164345 & 859.5 & 122.5 & 116.4 & 122.5 & 103.0 & $08-21-83$ & & & USGS \\
\hline 43 & 364554 & 1164855 & 987.5 & 97.8 & & & 74.5 & $01-11-87$ & & & USGS \\
\hline 61 & 364550 & 1164727 & 926.5 & 143.2 & & & 127.0 & $01-11-87$ & & & USGS \\
\hline 62 & 364514 & 1164829 & 981.4 & 115.8 & & & 108.1 & $01-01-87$ & & & USGS \\
\hline 63 & 364300 & 1163714 & 792.4 & 147.5 & & & & $01-11-87$ & D & & DALE, W. \\
\hline 64 & 364247 & 1164423 & 829.0 & 579.1 & 585.2 & 588.2 & 92.4 & $01-11-87$ & & & USGS \\
\hline 65 & 364246 & 1164457 & 829.0 & 426.7 & & & 85.8 & $01-11-87$ & & & USGS \\
\hline 66 & 364243 & 1164324 & 822.9 & 566.9 & 524.2 & 566.9 & 92.8 & $01-11-87$ & & & USGS \\
\hline 67 & 364130 & 1164112 & 800.9 & 292.6 & 283.5 & 286.5 & 82.2 & $08-03-86$ & & NA-6 WELL BGMW-10 & USGS \\
\hline 68 & 364130 & 1164112 & 804.6 & 98.4 & 97.5 & 98.4 & 82.1 & $01-11-87$ & & & USGS \\
\hline 69 & 364105 & 1163026 & 831.1 & 140.2 & 97.5 & 140.2 & 103.6 & $06-30-92$ & & CIND-R-LITE WELL & CIND-R-LITE LAS VEGAS, NV \\
\hline 70 & 364044 & 1163753 & 773.5 & 54.3 & & & & $00-00-00$ & & & \\
\hline 71 & 363934 & 1162558 & 816.5 & 74.1 & & & & $00-00-00$ & & VH-2 & WASHBURN, RICHARD \\
\hline 72 & 363907 & 1162357 & 819.9 & 143.6 & 31.7 & 143.6 & 101.5 & $05-20-61$ & & & BOSSINGHAM, BEN \\
\hline 73 & 363858 & 1162541 & 804.6 & 23.8 & & & & $11-11-53$ & D & & WASHBURN, RICHARD \\
\hline 82 & 363825 & 1162433 & 804.3 & 228.6 & 109.7 & 236.8 & 88.4 & $02-20-64$ & & AIRPORT WELL & DOING, WARREN \\
\hline 83 & 363825 & 1162632 & 795.5 & 173.7 & 115.8 & 173.7 & 90.1 & $01-15-87$ & & & SHAW, JAMES H. \\
\hline 84 & 363815 & 1161759 & 931.4 & 243.8 & 224.0 & 243.8 & 205.4 & $07-21-62$ & & TW-5 & U.S. GOVT \\
\hline 85 & 363750 & 1162000 & & & & & & $00-00-00$ & & & \\
\hline 86 & 363749 & 1161302 & 1807.4 & 112.8 & & & 102.1 & $10-01-59$ & & & JOE FALLIN \\
\hline
\end{tabular}




\begin{tabular}{|c|c|c|c|c|c|c|c|c|c|c|c|}
\hline $\begin{array}{l}\text { Site } \\
\text { No }\end{array}$ & Lat & Long & $\begin{array}{l}\text { Land } \\
\text { Elev }\end{array}$ & $\begin{array}{l}\text { Total } \\
\text { Depth }\end{array}$ & $\begin{array}{l}\text { Perf } \\
\text { top }\end{array}$ & $\begin{array}{l}\text { ration } \\
\text { bottom }\end{array}$ & $\begin{array}{l}\text { Water } \\
\text { Level }\end{array}$ & $\begin{array}{l}\text { Measure } \\
\text { Date }\end{array}$ & $\begin{array}{l}\text { Note } \\
\text { O C }\end{array}$ & Site Name & Owner \\
\hline 87 & 363744 & 1162637 & 783.9 & 151.8 & & 151.8 & 77.8 & $07-12-62$ & & & WASHBURN, RICHARD \\
\hline 88 & 363740 & 1162639 & & & & & & $00-00-00$ & & & \\
\hline 89 & 363715 & 1162445 & 853.4 & 32.0 & & & & $00-00-00$ & & & NYE COUNTY LAND CO. \\
\hline 90 & 363709 & 1162646 & 775.7 & & & & 71.7 & $01-15-87$ & & & WASHBURN, RICHARD \\
\hline 92 & 363701 & 1162640 & 774.2 & 134.7 & 91.4 & 128.0 & 69.8 & $10-18-58$ & & & WASHBURN, RICHARD \\
\hline 94 & 363549 & 1163050 & 742.2 & 159.4 & 47.2 & 159.4 & 46.4 & $01-07-87$ & & & NYE COUNTY DEVELOPMENT CO. \\
\hline 98 & 363540 & 1162408 & 771.1 & 94.8 & 46.0 & 94.8 & 45.1 & $09-16-63$ & & & MASON, L. \\
\hline 102 & 363528 & 1162842 & & & & & & $00-00-00$ & & & \\
\hline 103 & 363527 & 1162925 & 744.0 & 106.7 & & & 47.1 & $01-16-87$ & & & \\
\hline 119 & 363437 & 1160108 & 1050.0 & 381.9 & 352.6 & 374.3 & 314.1 & $02-11-64$ & & ARMY 6A & U.S. GOVT. \\
\hline 135 & 363415 & 1160926 & 846.4 & & & & & $00-00-00$ & & & \\
\hline 138 & 363410 & 1162611 & 743.7 & 204.2 & 64.6 & 204.2 & 52.5 & $01-15-87$ & & & NYE COUNTY DEVELOPMENT CO. \\
\hline 161 & 363333 & 1160642 & 858.9 & & & 138.5 & & $04-29-64$ & W & WELLS-STEWART & WELLS STEW \\
\hline 163 & 363330 & 1160700 & 243.8 & & & & & $00-00-00$ & & PT OF ROCKS & HWY-STEWART \\
\hline 176 & 363303 & 1163001 & 722.6 & 221.0 & 30.5 & 221.0 & 2.1 & $02-15-55$ & & & MEETER, L. \\
\hline 178 & 363303 & 1163001 & 722.6 & 221.0 & 30.5 & 221.0 & 2.1 & $02-15-55$ & & & DELEE, MORRIS \\
\hline 179 & 363303 & 1163001 & 722.6 & 221.0 & 30.5 & 221.0 & 34.8 & $01-10-87$ & & & RECORDS, HENRY H. \\
\hline 210 & 363214 & 1161335 & 733.0 & 311.5 & 211.8 & 228.6 & 13.5 & $10-18-66$ & & AMARGOSA TRACER NO. 1 & U.S. GOVT \\
\hline 214 & 363213 & 1161338 & 732.2 & 205.7 & 189.0 & 205.7 & 12.5 & $11-21-66$ & & TRACER WELL 3 & USGS \\
\hline 228 & 363153 & 1162216 & 723.6 & 30.5 & & & 30.6 & $03-15-87$ & & IMV BENTONITE MINE WELL & \\
\hline 279 & 363026 & 1161604 & 710.1 & & & & 24.5 & $03-09-72$ & & & SPRING MEADOWS INC. \\
\hline 280 & 363026 & 1161604 & 710.1 & & & & 30.7 & $03-09-72$ & & & \\
\hline 305 & 362934 & 1162959 & 701.0 & 152.4 & & & 12.4 & $06-25-62$ & & & H DAVIS \\
\hline 309 & 362929 & 1160857 & 729.7 & 65.5 & & & 11.2 & $02-04-60$ & & CHERRY PATCH WELL & CLARK, HERSHEL \& ETAL \\
\hline 320 & 362905 & 1160923 & 730.0 & 41.1 & 27.7 & 41.1 & 10.7 & $10-29-84$ & & & MADAME \\
\hline 350 & 362738 & 1161049 & 720.8 & & 6.7 & & 4.8 & $06-09-62$ & & BUCK MINING WINDMILL WELL & BUCK MINING CO. \\
\hline 351 & 362736 & 1162857 & 691.9 & 536.7 & 591.0 & 594.0 & 17.0 & $01-11-87$ & & & USGS \\
\hline 352 & 362736 & 1162857 & 691.9 & 30.5 & 29.6 & 30.5 & 15.7 & 01-11-87 & & & USGS \\
\hline 379 & 362525 & 1162743 & 667.8 & 332.2 & 324.0 & 324.9 & 2.1 & $08-01-86$ & & NA-9 WELL & USGS \\
\hline 409 & 362250 & 1162651 & 664.4 & 512.0 & 502.9 & 505.9 & 23.5 & $10-02-87$ & & & \\
\hline
\end{tabular}




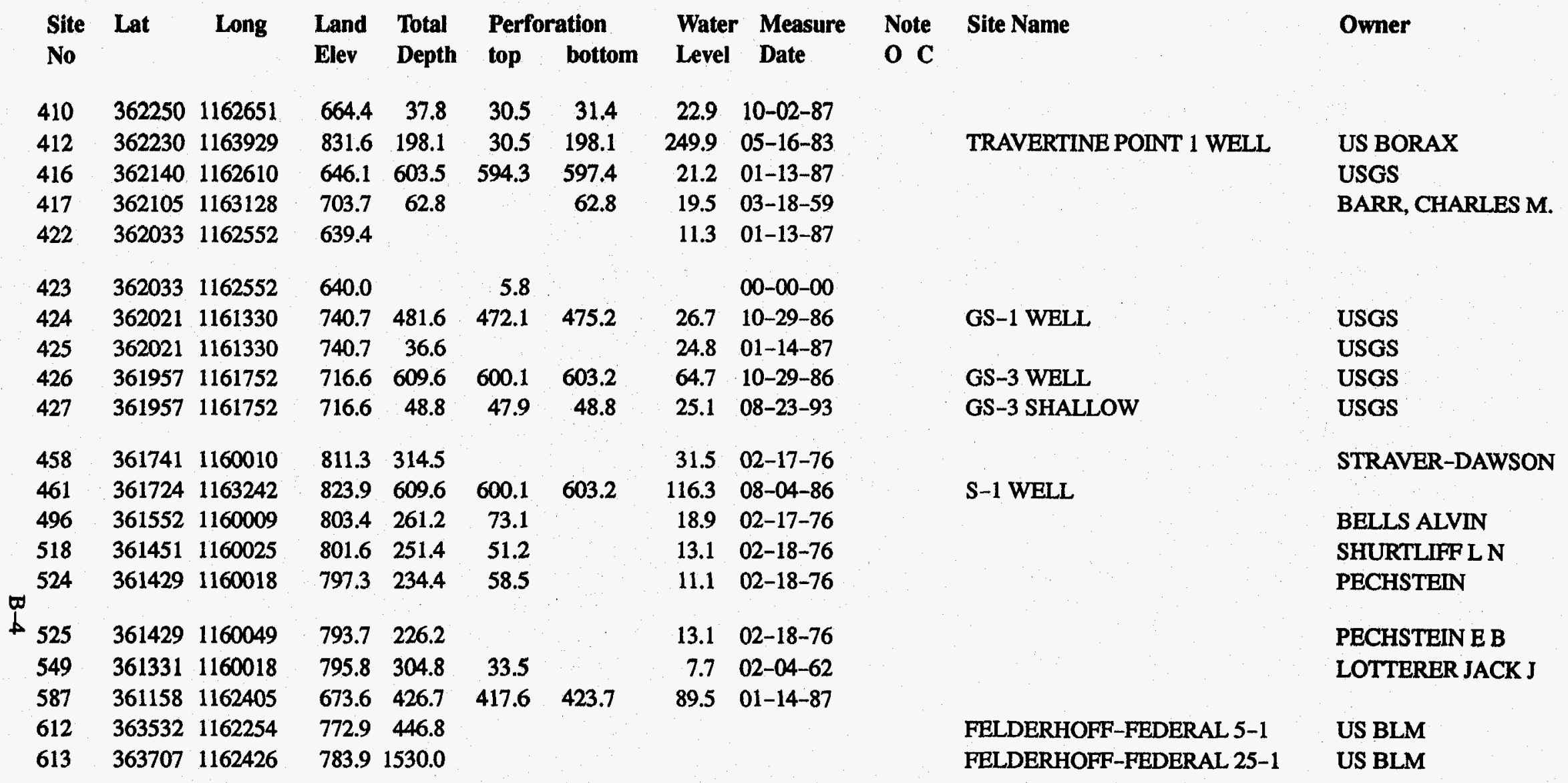




\section{DISTRIBUTION}

\section{U.S. Department of Energy}

Gylan Allen

Test Operations Division

Nevada Operations Office

U.S. Dept. of Energy

P.O. Box 98518

Las Vegas, NV 89193-8518

Doug Duncan

Hydrology Program Manager

Office of Environmental Restoration \& Waste

Management

Nevada Operations Office

U.S. Dept. of Energy

P.O. Box 98518

Las Vegas, NV 89193-8518

Don Elle, Director

Environment Protection Division

Nevada Operations Office

U.S. Dept. of Energy

P.O. Box 98518

Las Vegas, NV 89193-8518

Joseph N. Fiore, Acting Asst. Mgr.

Office of Environmental Restoration \& Waste

Management

Nevada Operations Office

U.S. Dept. of Energy

P.O. Box 98518

Las Vegas, NV 89193-8518

Joseph H. Kitchen

Technology and Program Management Division

Nevada Operations Office

U.S. Dept. of Energy

P.O. Box 98518

Las Vegas, NV 89193-8518

Steve Lawrence

Environmental Restoration Division

Nevada Operations Office

U.S. Dept. of Energy

P.O. Box 98518

Las Vegas, NV 89193-8518
John S. Ledbetter

Contracts Division

Nevada Operations Office

U.S. Dept. of Energy

P.O. Box 98518 , MS 505

Las Vegas, NV 89193-8518

Steve Leedom

Test Operations Division

Nevada Operations Office

U.S. Dept. of Energy

P.O. Box 98518

Las Vegas, NV 89193-8518

Steve Mellington, Director

Environmental Restoration Division

Nevada Operations Office

U.S. Dept. of Energy

P.O. Box 98518

Las Vegas, NV 89193-8518

Richard Pearl

Environmental Protection Division

Nevada Operations Office

U.S. Dept. of Energy

P.O. Box 98518

Las Vegas, NV 89193-8518

\section{U.S. Department of Defense}

David Bedsun

Defense Nuclear Agency

U.S. Department of Defense

P.O. Box 98539

Las Vegas, NV 89193-8539

\section{Lawrence Livermore National Laboratory}

Lee Davisson

Nuclear Chemistry Division

Lawrence Livermore National Laboratory

P.O. Box 808, MS L237

Livermore, CA 94550

Greg Nimz

Lawrence Livermore National Laboratory

P.O. Box 808, MS L233

Livermore, CA 94550 


\section{Los Alamos National Laboratory}

Joe Thompson

Los Alamos National Laboratory

INC-11, MS J514

P.O. Box 1663

Los Alamos, NM 87545

\section{U.S. Geological Survey}

Virginia Glanzman

U.S. Geological Survey

Box 2506, MS 913

Denver Federal Center

Denver, CO 80225

Randy Laczniak

U.S. Geological Survey

Water Resources Division

6770 S. Paradise Rd.

Las Vegas, NV 89119

Paul Orkild

U.S. Geological Survey

Box 2506, MS 913

Denver Federal Center

Denver, CO 80225

Doug Trudeau

U.S. Geological Survey

Water Resources Division

6770 S. Paradise Rd.

Las Vegas, NV 89119

\section{Desert Research Institute}

Roger Jacobson

Desert Research Institute

Water Resources Center

P.O. Box 19040

Las Vegas, NV 89132-0040

Marjory Jones

Desert Research Institute

Water Resources Center

P.O. Box 60220

Reno, NV 89506-0220
Dick French

Desert Research Institute

Water Resources Center

P.O. Box 19040

Las Vegas, NV 89132-0040

Reynolds Electrical \& Engineering Co.

Martha DeMarre

Chief, Document Research Section

Health Protection Dept.

Reynolds Electrical \& Engineering Co.

P.O. Box 98521

Las Vegas, NV 89193-8521

Brian Dozier

Reynolds Electrical \& Engineering Co.

2501 Wyandotte

Mercury, NV 89102

\section{State of Nevada}

Larry Franks

Nevada State Health Department

Radiological Health Section

620 Belrose Avenue

Las Vegas, NV 89158

\section{Libraries}

Annie Kelley

State Documents Department

Nevada State Library

Capitol Complex

Carson City, NV 89710

Archives

Getchell Library

University of Nevada, Reno

Beverly Carter

MacKay School of Mines Library

University of Nevada, Reno

Document Section, Library

University of Nevada, Las Vegas

4505 Maryland Parkway

Las Vegas, NV 89154

Southern Nevada Science Center

Water Resources Center, Library

P.O. Box 19040

Las Vegas, NV 89132-0040 
Stead Library

Desert Research Institute

Reno, Nevada

Technical Information Resource Center

Nevada Operations Office

U.S. Dept. of Energy

P.O. Box 98518

Las Vegas, NV 89193-8518

Water Resources Research Archives

University of California

Room 40, North Gate Hall

Berkeley, CA 94720
International Technology Corp.

Rick Waddell

Geotrans

c/o IT

4330 Valley View

Suite 112

MS -439

Las Vegas, NV 89103

Joe Yeasted

International Technology Corp.

4330 S. Valley View

Suite 114

Las Vegas, NV 89132-0040 